\title{
1 Utilisation of staphylococcal immune evasion protein Sbi as a novel
}

4 Yang, Y. \$1, Back, CR. $\$ 1$, Gräwert, MA. ${ }^{2}$, Wahid, AA. ${ }^{1}$, Denton, H. ${ }^{3}$, Kildani, R. ${ }^{3}$, Paulin, J. ${ }^{3}$,

5 Wörner, K. ${ }^{4}$, Kaiser, W. ${ }^{4}$, Svergun, DI. ${ }^{2}$, Sartbaeva, A. ${ }^{5}$ Watts, AG. ${ }^{6}$, Marchbank, KJ. ${ }^{3 *}$, and 6 van den Elsen, JMH ${ }^{1 *}$.

8 1Department of Biology and Biochemistry, University of Bath, Bath, UK

9 2European Molecular Biology Laboratory, Hamburg Unit

10 c/o Deutsches Elektronen-Synchrotron, Hamburg, Germany

11 Institute of Cellular Medicine, Newcastle University, Newcastle-upon-Tyne, UK

12 'Dynamic Biosensors GmbH, Martinsried, Germany

13 5Department of Chemistry, University of Bath, Bath, UK

14 6Department of Pharmacy and Pharmacology, University of Bath, Bath, UK

15

16

17

18

19

20

21

22

23

24

25

26

27

28

29

30

31

32

33

34

35

36

37
$\S$ Contributed equally to this work

*Co-Corresponding author addresses: Jean van den Elsen (j.m.h.v.elsen@bath.ac.uk) and Kevin James Marchbank (Kevin.marchbank@ncl.ac.uk) 


\section{Abstract}

Co-ligation of the B cell antigen receptor with complement receptor 2 on B-cells via a C3d-opsonised antigen complex significantly lowers the threshold required for B cell activation. Consequently, fusions of antigens with C3d polymers have shown great potential in vaccine design. However, these linear arrays of C3d multimers do not mimic the natural opsonisation of antigens with C3d. Here we investigate the potential of using the unique complement activating characteristics of Staphylococcal immune-evasion protein Sbi to develop a

50 pro-vaccine approach that spontaneously coats antigens with C3 degradation

51 products in a natural way. We show that Sbi rapidly triggers the alternative

52 complement pathway through recruitment of complement regulators, forming a

53 tripartite complex that acts as a competitive antagonist of factor $\mathrm{H}$, resulting in

54 enhanced complement consumption. These functional results are corroborated

55 by the structure of this complement activating Sbi-III-IV:C3d:FHR-1 complex.

56 Finally, we demonstrate that Sbi, fused with Mycobacterium tuberculosis antigen

57 Ag85b, causes efficient opsonisation with C3 fragments, thereby enhancing the 58 immune response significantly beyond that of Ag85b alone, providing proof of 59 concept for our pro-vaccine approach. 


\section{Introduction}

61 Opsonisation of an antigen with $\mathrm{C} 3 \mathrm{~d}(\mathrm{~g})$, the final degradation product of

62 complement component $\mathrm{C} 3$, results in the co-ligation of the $\mathrm{B}$ cell antigen

63 receptor and complement receptor 2 (CR2) on B cells, thereby instigating a

64 profound molecular adjuvant effect, i.e. this co-ligation of receptor complexes

65 lowers the threshold of antigen required for B cell activation by up to 10,000 fold

66 [1-3]. Furthermore, as CR2 is also expressed highly on follicular dendritic cells

67 (FDCs) [4] the presence of $\mathrm{C} 3 \mathrm{~d}(\mathrm{~g})$ on the antigen allows it to be trafficked onto

68 and trapped at the surface of these cells [5]. This provides an essential depot of

69 antigen to support the germinal center reaction and maintain the ongoing

70 immune response including the generation of high affinity antibodies and

71 memory B-cells $[3,4,6]$. B cells can also have an important role as antigen

72 presenting cells (APCs) [7, 8] and have been shown to contribute to T-helper cell

73 priming $[9,10]$ and therefore, antigen-C3d-CR2 interactions play a key role in

74 humoral immunity [5]. Additionally, C3d activation of $\mathrm{T}$ helper cells has also

75 been described in a CR2 independent manner [11], underlining the importance

76 of C3d opsonisation in stimulation of the immune system to respond.

77 Not surprisingly, this functionality led to the idea that recombinant versions

78 of C3d would make an ideal natural adjuvant and to the subsequent design of

79 linear polymers of human C3d [12]. Indeed, these linear arrays of C3d multimers

80 (3-mer to 20-mer) when fused directly to an antigen can act as potent activators

81 of human B-cells. However, they do not mimic the natural opsonisation of 
82

83

84

antigens by C3d at a molecular level and do not always enhance immune responses [13]. After activation of $\mathrm{C} 3, \mathrm{C} 3 \mathrm{~b}$ attaches directly to the antigen surface via the reactive thioester on the convex face of the protein's thioester domain (TED). In the presence of complement regulators (factor I (FI) and its co-factors, such as factor $\mathrm{H}(\mathrm{FH})$ and $\mathrm{CR} 1$ ) this is rapidly converted to $\mathrm{iC} 3 \mathrm{~b}$ and then to C3d, exposing the concave CR2 binding site of the TED fragment away from the antigen surface [14]. It is likely that multiple iC3b/C3d molecules attach to complex antigens/pathogen surfaces during the initial activation phases of complement, creating high-avidity binding site for complement fragment receptors.

In the last two decades, structural biology has helped to unveil many of the molecular aspects that are crucial for the activation and regulation of the complement system. Most notable are the crystal structures of the central complement component activation states, native C3 [15], activated C3b and inactive C3c [16]. The structure of C3b in complex with factors B and D [17] subsequently revealed a detailed view of the alternative pathway C3 convertase assembly and its activation, leading to the amplified cleavage of C3 molecules that result in opsonisation and clearance of microbial pathogens and host debris. The covalent attachment of $\mathrm{C} 3 \mathrm{~b}$ to surfaces does not discriminate between self or non-self surfaces and requires tight regulation to protect host surfaces. Structures of C3b in complex with FH domains 1-4 [18] and domains 19-20 [19, 20] provided insights into protection of host cells [21] and demonstrated how 
104 factor H-related proteins (FHRs) function as competitive antagonists of $\mathrm{FH}$,

105 modulating complement activation and providing improved discrimination of

106 self and non-self surfaces [22]. The subsequent structure of the complex of C3b,

$107 \mathrm{FH}_{1-4}$ and regulator factor I [23] improved our understanding in the proteolytic

108 cleavage of $\mathrm{C} 3 \mathrm{~b}$ to the late-stage opsonins $\mathrm{iC} 3 \mathrm{~b}$ or $\mathrm{C} 3 \mathrm{dg}$ and provided the basis

109 for the regulator-dependent differences in processing and immune recognition

110 of opsonized material.

111 Here we investigate the potential to harness the unique

112 complement-stimulatory characteristics of Staphylococcus aureus

113 immunomodulator Sbi to develop 'pro-vaccines'. Sbi components would trigger

114 natural complement activation in the host and coat antigen surfaces with

115 complement component C3 degradation products, thereby enhancing the degree

116 of immunogenicity of target antigens. Research from our lab previously revealed

117 that Sbi contains two domains (III and IV), which bind to the central complement

118 component C3 and cause futile fluid phase consumption of this component [24].

119 Therefore, these two domains of Sbi offer the potential to not only coat an

120 antigen with the natural adjuvant C3d, but also to generate anaphylatoxins and

121 the full range of C3 opsonins. Such an approach has the clear potential to activate

122 many immune cells unlike recombinant C3d fragment-based adjuvants of the

123 past, that, due to the restricted expression pattern of CR2, were largely focused

124 to B cells. Furthermore, the direct activation of complement close to the target

125 antigen (with the associated anaphylatoxin generation) may be critical for 
bioRxiv preprint doi: https://doi.org/10.1101/413294; this version posted September 10, 2018. The copyright holder for this preprint (which

was not certified by peer review) is the author/funder, who has granted bioRxiv a license to display the preprint in perpetuity. It is made available under aCC-BY-NC-ND 4.0 International license.

126 generating appropriate inflammatory immune responses, both humoral and

127 cellular; needed to immunise against complex pathogenic targets.

128 In this study we first investigate the molecular mode of action of Sbi-III-IV

129 and evaluate the importance of the tripartite complex formation between Sbi,

$130 \mathrm{C} 3 \mathrm{~d}$ and complement regulators factor $\mathrm{H}(\mathrm{FH})$ or factor H-related proteins (FHRs)

131 for complement activation. Based on these findings, we then tested whether our

132 pro-vaccine strategy would be successful by using Mycobacterium tuberculosis

133 antigen 85b (Ag85b) as a model antigen in a fusion construct containing Sbi

134 domains III and IV. We show that this Sbi-Ag85b conjugate is opsonized by C3

135 degradation products in serum, and when administered to mice, leads to an

136 enhanced immune response in vivo, but only in mice that possess C3 and

137 complement receptor 1 and 2, demonstrating proof of concept for this adjuvant

138 compound. 


\section{Results:}

141 Sbi-III-IV triggers C3 consumption via activation of the alternative

\section{2 complement pathway, forming a covalent adduct with $\mathrm{C} 3 \mathrm{~b}$}

143 To investigate the molecular details of the C3 futile consumption caused by

144 Sbi, a protein construct consisting of domains III and IV (Sbi-III-IV) was

145 incubated with normal human serum (NHS) and analyzed using western blotting.

146 As seen previously [24], we found that Sbi-III-IV-induced C3 consumption results

147 in the deposition of metastable C3b molecules onto serum proteins, causing the

148 formation of high molecular weight C3b covalent adduct species with serum

149 proteins (Figure 1a). Immuno-blotting analyses using a polyclonal anti-Sbi

150 antibody (Figure 1b) reveals that a small fraction of Sbi-III-IV molecule also

151 forms a covalent adduct with a nascent C3b molecule that is subsequently

152 converted into a smaller Sbi-iC3b adduct as a result of proteolytic processing by

153 serum proteases. In addition, we show that Sbi-III-IV-induced C3 consumption

154 coincides with the release of the C3a anaphylatoxin fragment (Figure 1c), and the

155 proteolytic activation of factor B (FB) (Figure 1d), confirming the alternative

156 complement pathway as the driving force behind this process. Pre-incubation of

157 serum with Sbi-III-IV results in the loss of serum hemolytic ability caused by the

158 futile consumption of fluid C3 (Figure 1e). Without pre-incubation, the Sbi-III-IV

159 construct does not protect rabbit erythrocytes from lysis in NHS under AP 160 conditions. 
bioRxiv preprint doi: https://doi.org/10.1101/413294; this version posted September 10, 2018. The copyright holder for this preprint (which was not certified by peer review) is the author/funder, who has granted bioRxiv a license to display the preprint in perpetuity. It is made available under aCC-BY-NC-ND 4.0 International license.

161

162

163

164

165

166

167 178 (Table 2 and Supplementary Figure S2B). A more detailed structural analysis of 179 these conformational changes follows below.

\section{Sbi domain III residue $\mathrm{K173}$ is essential for complement consumption}

In order to gain understanding of the individual roles of Sbi domains III and IV in AP activation, a systematic site-directed mutagenesis approach was used, mainly focusing on charged and polar amino acids (for details see Supplementary Table S1 and Figure S1). Functional screening of these mutants identified K173, located within Sbi domain III (Figure 1f), as an essential contributor to triggering C3 consumption. Sbi mutant K173A shows no complement activation after 30 minutes incubation with human serum, demonstrating a comparable complement activation defect to the previously identified C3d binding mutant R231A [24, 25] (Figure 1f and g), located in Sbi domain IV. Assessment of the C3d binding affinity, using switchSENSE (Table 1 and Supplementary Figure S2A), shows that contrary to R231A the C3d binding capacity of $\mathrm{K} 173 \mathrm{~A}$ is unaffected, indicating it is essential for the role for domain

III in the futile consumption of C3. Interestingly, our switchSENSE analyses of the C3d binding characteristics also shows a reduced hydrodynamic diameter for K173A compared to WT and the C3d impaired binding mutant R231A, indication that this mutation in domain III results in a more compact Sbi:C3d complex 
183 combination with the C-terminal part of $\mathrm{FH}\left(\mathrm{FH}_{19-20}\right)$, forming tripartite 184 complexes [26]. Many FHRs share SCR modules with high $\mathrm{FH}_{19-20}$ sequence 185 identity [22] particularly FHR1 which has been demonstrated to have significant 186 complement dysregulation potential [21]. Thus, we investigated the potential 187 role for Sbi-III-IV in mediating the formation of tripartite complexes with C3 188 fragments and FHR-1, FHR-2 or FHR-5.

On a C3b opsonised surface plasmon resonance (SPR) sensor chip, the 190 presence of wild-type Sbi-III-IV clearly enhanced the binding of FH, FHR-1, 191 FHR-2, FHR-5 as well as $\mathrm{FH}_{19-20}$ (at fixed concentrations of 100, 12.5, 20, 25 and 192 20nM, respectively) to the surface in a concentration dependent manner (Figure 193 2a). However, in the case of the K173A mutant, tripartite complex formation 194 with FH or FHR-1, 2, 5 or $\mathrm{FH}_{19-20}$ is significantly impaired, showing decreased 195 binding and more rapid dissociation compared to WT Sbi-III-IV (Figure 2b and 196 2c). We also co-injected Sbi-III-IV with FH or FHR-1, flowing opsonized iC3b or 197 amine-coupled C3d(g) across the surface. On these surfaces, the fold-changes in 198 FH (or FHR-1) binding levels were also enhanced even at reduced Sbi-III-IV 199 concentration (Supplementary Figures S3A-D). Sbi-III-IV acts as a competitive antagonist of FH via the recruitment of FHRS and 5 binding to the $\mathrm{C} 3$ activation fragment $\mathrm{C} 3 \mathrm{~b}$ and late-stage proteolytic 204 fragments iC3b and C3d(g). To further our understanding of the mechanism of 
205 FH or FHR recruitment and the contribution of these tripartite complexes to AP 206 complement activation, we used a rabbit erythrocyte haemolytic assay. In the 207 presence of Sbi-III-IV and endogenous FH (and FHRs), in NHS, addition of 208 recombinant FHR-1 or FHR-2 resulted in significantly enhanced C3 consumption 209 (Figure 3a), as evidenced by the reduction in erythrocyte lysis in a concentration 210 dependent manner. In the absence of Sbi-III-IV only baseline C3 consumption 211 was observed. Although FHR-5 alone can reduce erythrocyte lysis in a 212 concentration dependent manner, as described previously [27], in the presence 213 of Sbi-III-IV C3 consumption by FHR-5 is clearly enhanced (Figure 3b). As 214 predicted, the results in Figure $3 c$ indicate that the observed reduction in 215 erythrocyte lysis caused by C3 fluid phase consumption, in the case of FHR1 and 216 likely the remaining FHRs, is mediated by the C-terminal SCR domains of the 217 protein rather than the N-terminal domains.

218 Whilst our SPR and rabbit erythrocyte assay clearly indicate that in vitro 219 Sbi-III-IV can recruit FHRs in tripartite complexes with C3b and thereby enhance 220 fluid phase complement consumption, it has to be taken into account that the 221 physiological molar concentrations of FHR-1, 2 and 5 are 13-164 fold less than 222 that of $\mathrm{FH}[21,28]$. To further investigate the potential competitive binding 223 between FH and the FHRs in Sbi-III-IV mediated tripartite complexes, we used an 224 ELISA-based assay where we applied FH (25 nM) and Sbi-III-IV (1 $\mu \mathrm{M}$, in the 225 presence of a concentration range of FHRs (9.3 - 150nM)) onto a C3b coated 226 plate. Subsequently, we assessed the percentage of FH bound using monoclonal 
227 antibody OX-24. Figure 3d shows that FHR-1 can compete with FH to bind C3b,

228 decreasing the percentage of residual $\mathrm{FH}$ bound to $\mathrm{C} 3 \mathrm{~b}$ from $\sim 70 \%$ at the lowest

229 FHR-1 concentration to $\sim 30 \%$ at the highest concentration. In the presence of

230 Sbi-III-IV WT this effect is dramatically increased with only $\sim 25 \%$ residual FH

231 bound at the lowest FHR-1 concentration, reducing to $\sim 0 \%$ at the highest FHR-1

232 concentration (Figure 3e). These results clearly indicate that Sbi-III-IV can

233 preferentially recruit FHR-1 to form a tripartite complex with C3b. Similarly,

234 enhancement of recruitment was observed with FHR-5 and fragment $\mathrm{FH}_{19-20}$ but

235 only weakly with FHR-2. Although unable to activate complement, Sbi-III-IV

236 mutant K173A is still able to compete for the binding of FHR-1 in the presence of

$237 \mathrm{FH}$, but its ability to enhance binding of FHR-2 and FHR-5 to C3b is clearly

238 affected (Figure 3f).

239 To assess the potential AP de-regulatory roles of the Sbi-III-IV mediated

240 tripartite complexes. We subjected them to a novel C3 convertase decay

241 acceleration activity (DAA) assay and a fluid phase C3b co-factor activity (CFA)

242 assay $[29,30]$. We demonstrated that in absence of Sbi, FHR-1 failed to

243 antagonise FH efficiently and show a difference in the level of C3 convertase

244 formation (Figure 3g). Co-injection of FHR-1 and -5 shows reduced C3

245 convertase formation, which is in accordance with the results from a previous

246 study [31]. However, the presence of Sbi $(2 \mu \mathrm{M})$ potentiates the $\mathrm{FH}$ antagonising

247 effect of FHR-1, and to a lesser extent that of FHR-5, at a physiologically relevant

248 concentration ratio (FH 2000 nM: FHR-1 200 nM : FHR-5 20 nM), resulting in 
bioRxiv preprint doi: https://doi.org/10.1101/413294; this version posted September 10, 2018. The copyright holder for this preprint (which was not certified by peer review) is the author/funder, who has granted bioRxiv a license to display the preprint in perpetuity. It is made available under aCC-BY-NC-ND 4.0 International license.

249 increased C3 convertase formation on a C3b surface (Figure 3h). The baseline 250 C3b breakdown rate was acquired in the presence of $\mathrm{FH}(0.160 \mu \mathrm{M})$ and FI $251(0.017 \mu \mathrm{M})$, and subsequent measurements were performed in the presence of 252 FHR alone $(0.32 \mu \mathrm{M})$ and in combination with Sbi-III-IV $(1 \mu \mathrm{M})$. As shown in 253 Figure 3i and Supplementary Figure S3F, the presence of FHR-1, 2 or 5 increases 254 the C3b fluid phase half-life to different degrees, with FHR-5 showing the largest 255 increase in half-life. Most interestingly, the C3b half-life could be further 256 extended by the addition of Sbi-III-IV.

The Sbi-III-IV:C3d:FHR-1 tripartite complex forms a dimer in solution

To investigate the structural characteristics of the Sbi-III-IV:C3d:FHR1 260 tripartite complex, we used small angle X-ray scattering (SAXS). The scattering 261 profile collected at an equimolar mixing ratio is shown in Figure 4 in log plot (a) 262 as well as Kratky plot (b). The featureless descend in the log plot and the plateau 263 in the latter is characteristic for scattering of particles that are, at least partially, 264 disordered.

265 The SAXS data and the overall parameters obtained (Supplementary Table 266 S2) suggest that the complex is largely dimeric but rather flexible in solution. 267 Quantitative flexibility analysis was performed using the ensemble optimisation 268 method EOM [32], which fits the experimental data using scattering computed 269 from conformational ensembles. Models with randomized linkers were generated based on the known structures of FHR-11-2 (3zd2, [21]); FH18-20 (3sw0, 
271 [33]), containing the equivalent of FHR-13; FH $19-20: \mathrm{C} 3 \mathrm{~d}$ complex (2xqw, [20]),

272 corresponding with FHR-14-5; and the Sbi-IV:C3d complex (2wy8, [34]). To

273 account for the dimerisation, P2 symmetry was applied, using the FHR-1 $1_{1-2}$ dimer

274 interface as seen in the crystal structure (3zd2). The distributions of the overall

275 parameters in the selected structures compared with those of the original pool

276 (Figure 4c) suggests that the complex is rather flexible with a slight preference

277 for extended structures in solution. The subset of most typical models (and the

278 volume percentage of their contribution) shown in Figure 4c indicate that in

279 addition to the expected contact sites with C3d, Sbi-III domain appears to also 280 interact with FHR-1, corroborating the functional results described above. Figure $2814 d$ shows a schematic representation of the dimeric Sbi-III-IV:C3d:FHR-1 282 complex observed in solution.

\section{K173A restricts the conformational freedom of Sbi domain III}

To examine the possible structural effects of the K173A substitution in Sbi

286 domain III, SAXS data was collected on the Sbi-III-IV(K173A):C3d complex and 287 compared to the wild-type Sbi-III-IV:C3d complex published previously [34]. The 288 experimental scattering pattern collected at $240 \mu \mathrm{M}(\sim 12 \mathrm{mg} / \mathrm{ml})$ is presented 289 in Figure 4e and the structural parameters derived from this data are given in 290 Supplementary Table S2. The estimated molecular mass (MM) of the solute 291 agrees within the errors with the values predicted for a 1:1 complex $(\sim 15 \mathrm{kDa}+$ $29235 \mathrm{kDa}$ ). At lower concentration a decrease in the MM estimates is observed 
293 which suggests that the complex slowly begins to dissociate. The previously 294 described wild-type Sbi-III-IV:C3d data on the other hand, suggests that at higher 295 concentrations, higher oligomeric species are present, thus, for the comparison 296 here, data collected at $0.6 \mathrm{mg} / \mathrm{ml}$ is shown. The faster descend of the wild-type 297 data, which translates to a larger $\mathrm{Rg}$, suggests that rearrangements of the flexible 298 N-terminus lead to a more elongated particle (Rg wild-type $=32.8 \AA$ ) as 299 compared to $\mathrm{K} 173 \mathrm{~A}$ mutant $(\mathrm{Rg} \mathrm{K} 173 \mathrm{~A}=30.6 \AA)$. This is in strong agreement 300 with the switchSENSE analysis of the C3d binding characteristics, which show a 301 reduced hydrodynamic diameter for K173A compared to WT (Table 2). The $a b$ initio low resolution models of the complex reconstructed from the 303 highest concentration data using DAMMIF [35] showed a large cone shaped 304 molecule with a volume of $124 \mathrm{~nm}^{3}$ (Figure 4f). The resolution of the 305 reconstruction is estimated to be $29+/-2 \AA$ [36]. The large base of the cone can 306 accommodate the crystal structure of Sbi-IV:C3d complex (2wy8) [34]. The extra 307 space at the tip of the cone would be sufficient to harbor the $60 \mathrm{~N}$-terminal 308 residues comprising the Sbi-III domain. A more detailed modeling was 309 conducted with the program Coral [37], utilizing the available high-resolution 310 model of Sbi-IV:C3d and allowing for 60 additional beads to be added that mimic 311 the missing Sbi-III domain. Twenty independent Coral runs were performed 312 which all yielded models with a more or less structured N-terminal region, 313 suggesting that Sbi-III-IV(K173A) in complex with C3d is conformationally 314 restricted compared to wild-type Sbi-III-IV. This is further supported by the 
316 Surprisingly, whilst repeating these analyses using a proposed alternative

317 binding mode of the Sbi-IV:C3d complex (represented by 2wy7), where Sbi-IV is

318 seen bound at the convex face of $\mathrm{C} 3 \mathrm{~d}$, the $\chi^{2}$ value is greatly improved

319 (Supplementary Figure S4C). With this modeling approach a similar restricted 320 conformation is observed for the N-terminus of Sbi-III-IV(K173A). Further 321 studies are currently being conducted to further investigate the potential 322 physiological relevance of this alternative Sbi-IV:C3d binding mode.

\section{A fusion construct of Sbi-III-IV with M. tuberculosis Ag85b activates the AP}

To test the potential of Sbi-III-IV to induce C3d opsonisation in a vaccine

S5A). Based on the SAXS structure of the Sbi-III-IV:C3d:FHR-1 tripartite complex,

331 long flexible linker between Sbi-IV and Ag85b to ensure accessibility and

332 flexibility of the functional domains. Expressed and purified fusion protein was

333 subsequently structurally and functionally characterized.

334 Circular dichroism analysis of the Sbi-III-IV-Ag85b fusion indicates that the protein construct is folded and that the secondary structural elements of both

336 parent proteins have been preserved (Supplementary Figure S5B). SAXS data 
337 obtained for the fusion protein demonstrate that both the Ag85b domain as well

338 as Sbi-IV domain are accessible (Figure 5b and Supplementary Figure S5C).

Functional activity of the Sbi-III-IV-Ag85b fusion construct was assessed using an AP complement activity assays (WIESLAB, Euro Diagnostica), showing

341 strong C3 depletion activity (Supplementary Figure S5D), whilst Ag85b on its 342 own showed no complement activating properties. These results confirm that 343 the complement activating properties of Sbi III-IV are not impaired as part of the 344 fusion construct. The western blot analyses presented in Figure $5 \mathrm{c}$ and 345 Supplementary Figure S5E confirm these results, showing both C3 activation and opsonisation by the Sbi-III-IV-Ag85b fusion construct when incubated with NHS.

347 Interestingly, C3 activation and consumption occur more rapidly with the fusion 348 construct when compared to Sbi-III-IV (Figure 1a and 1b) under the same 349 conditions. Whilst Sbi-III-IV shows opsonisation with a single molecule of C3b 350 (Figure 1b), the Sbi-III-IV-Ag85b fusion is opsonized by 2 molecules of C3b that 351 over time degrade to iC3b and C3d (Figure 5c and Supplementary Figure S5E). 352 Interestingly, opsonisation of Ag85b with C3 fragments also occurs when 353 co-incubated with Sbi-III-IV in NHS.

\section{Sbi-III-IV acts as an adjuvant in mice when immunized with Ag85b}

Based on the ability of Sbi-III-IV to activate complement (Figures 1b, 1c

357 (human serum) and 6a (mouse serum)) and opsonise Ag85b with complement 358 C3 break down fragments (Figure 5c), we expected that this new fusion protein 359 when injected into mice would elicit a greater immune response to the 
360 Sbi-III-IV-Ag85b fusion protein than Ag85b administered alone (in PBS). Indeed,

361 wild-type C57bl/6 mice immunized I.P. (or I.V., data not shown) with

362 Sbi-III-IV-Ag85b generated a greater than 4 fold increase in immune response

363 initially and following the boost when compared to Ag85b alone (Figure 6b).

364 Furthermore, when mice were immunized with a mixture of Sbi-III-IV and Ag85b

365 (not fused together), this also resulted in a significantly improved immune

366 response, corroborating the role of C3 fragment opsonisation of the antigen in

367 this process (see Supplementary Figure S5E). Subsequent, studies using C3\% and

$368 \mathrm{Cr} 2 \%$ mice clearly demonstrated that C3 and C3 breakdown fragment receptors

369 (CR1 and CR2) were essential for this "adjuvant" function, respectively (Figure

370 6c). Overall, these data clearly suggest that complement AP dysregulation

371 function of the Sbi-III-IV domain can be harnessed to improve immune responses

372 through the coating of antigens with C3 breakdown fragments. 
373

375

multiple copies of $\mathrm{C} 3 \mathrm{~d}$, in a linear trimer, could enhance antigen-specific

390 responses up to 10,000 fold [3]. However, the initial potential of trimeric C3d, as

391 a highly potent molecular adjuvant, has not been realized and the reason(s) for

392 this remain(s) unclear. One possible explanation is that the artificial linear 393 trimer structure fails to represent naturally opsonised antigen, and consequently

394 does not provide sufficient CR cross-linking or additional inflammatory signals 
395 for the B cell (or APC) activation threshold to be reached. One possible approach to overcome this is attaching more C3d to test antigens, but that approach is also

397 limited [42]. In the light of these and other findings [11, 13, 43], we considered 398 that with understanding of the mode of action of Sbi-III-IV we might be able to 399 develop a new complement activation based immune adjuvant.

400 The first clue to a mechanism for Sbi's ability to rapidly activate the AP came 401 from monitoring Sbi-III-IV treated NHS in a time course using anti-C3 and 402 anti-Sbi immuno-blotting. Here, we demonstrated that metastable C3b not only 403 attaches covalently to serum proteins but also to Sbi-III-IV itself; as a 404 transacylation target (Figure 1). This makes sense in the respect that Sbi's 405 affinity to C3 obviously places it in close proximity to the site of complement 406 turnover and we speculate that C3b deposited on Sbi-III-IV could help extend the 407 fluid phase half-life of C3b, preventing FH and FI from binding and inactivating 408 as normal, perhaps similar to covalent adducts of C3b with IgG $[44,45]$. However, as we have shown previously, Sbi-III-IV also interacts with 410 complement regulators $\mathrm{FH}$ and FHR-1, in addition to binding $\mathrm{C} 3 \mathrm{~b}$ and its 411 degradation products, thereby forming tripartite complexes [26]. We next 412 investigated whether Sbi-III-IV acts as a competitive antagonist of FH via the 413 recruitment of FHR-1 and 5 into tripartite complexes and that FHR-1 can 414 effectively displace FH from the tripartite complex. To this end, data from our 415 systematic site-directed mutagenesis screen brought to light several Sbi mutants 416 with complement activation defects (Figure 1, Supplementary Figure S1). For 
417 instance, we demonstrated that an alanine substitution in Sbi domain III at

418 position 173 resulted in a dramatic reduction in C3 consumption activity (Figure

419 1g). Notably, although a similar effect was observed with a previously identified

420 mutation in domain IV with impaired C3d binding (R231A), K173A showed only

421 slightly impaired C3d binding capacity (Table 1) suggesting a different

422 mechanism. We therefore postulated that the K173A mutant would be ideal to

423 elucidate the structural and functional role of Sbi domain III in the activation of

424 complement and found that K173 in Sbi domain III was crucial for the

425 recruitment of FHR-5 and that the K173A mutation only slightly affects FHR-1

426 binding (Figures 2 and 3). These findings implicate a direct role of Sbi domain III

427 in the tripartite complex formation with these FHRs and that this likely occurs

428 via interactions with the C-terminal SCR domains that share sequence identity

429 with $\mathrm{FH}_{19-20}$. We confirm this by showing that increasing concentrations of

430 recombinant FHR-1, FHR-2, FHR-5 and $\mathrm{FH}_{19-20}$ in serum indeed potentiate

431 Sbi-III-IV mediated C3 consumption, whilst the N-terminal SCRs (FHR-1 1 -2) fails

432 to do this (Figure 3).

433 We also observed that Sbi greatly enhances the binding of FHRs to C3b,

434 thereby antagonizing FH activity, as shown by the C3 convertase decay

435 accelerating activity (DAA) assay (Figure $3 \mathrm{~g}$ and $\mathrm{h}$ ). These results imply that the

436 FHR-1 or FHR-5 containing tripartite complexes can protect the AP C3

437 convertase, aiding the consumption of $\mathrm{C} 3$. These findings further enhance the

438 notion that the FHR family has diversified AP de-regulatory functions, where 
439 FHR-1 seems more efficient in counteracting the DAA of FH, whilst in contrast

440 FHR-5 potently antagonizes the cofactor activity (CA) of FH. The observed

441 Sbi-III-IV mediated shift in the complement regulatory balance towards C3

442 activation could potentially be further enhanced by the formation of

443 homo/heterodimeric forms of FHR-1 with itself and with other FHRs (FHR-2 and

444 FHR-5) [12]. These data link to an ongoing evolutionary 'arms race' where FH

445 was initially hijacked by $S$. aureus to protect it from complement [46] and then

446 FHRs (devoid of intrinsic complement regulatory activity) were

447 evolved/deployed by the host to compete with FH on that surface and restore

448 complement opsonisation of the pathogen [22]. Perhaps the release/secretion of

449 Sbi from $S$. aureus is a more recent event in this arms race with the host, which

450 takes the $\mathrm{C} 3 \mathrm{~b} / \mathrm{C} 3$ convertase binding potential away from the bacterial surface

451 and leads to local rapid fluid phase consumption of complement, i.e. local

452 decomplementation and bacterial survival/propagation. Our understanding of

453 the role and complexity of FHRs in immune evasion strategies is still in its

454 infancy [46], but this study underlines the potency of another strategy in this

455 process.

456 Using FHR-1 as a 'model' dimerization domain containing FHR, structural

457 analysis of the Sbi-III-IV:C3d:FHR-1 tripartite complex, using SAXS, indeed

458 suggests the formation of a dimer mediated by FHR-1 domain 1 and 2 and

459 provides details of the role of the extended unfolded nature of domain III in the

460 binding of FHR-1 (Figure 4). The molecular basis of the preferential binding of 
461 FHR-1 over FH cannot easily be explained on the basis of differences in amino

462 acid sequence between the two complement regulators, since their C3d binding

463 regions (SCR 4-5 of FHR-1 and SCR 19-20 of FH) share 99\% sequence identity.

464 However, our SAXS analyses, and binding studies using $\mathrm{C} 3 \mathrm{~d}(\mathrm{~g})$ or $\mathrm{iC} 3 \mathrm{~b}$ as ligands

465 (Figures 3 and 4), indicate that the C-terminal regions of FHR proteins are

466 readily exposed, unlike those of FH that exist in a "latent" conformation with the

467 C-terminal part of the protein folded back and partially blocked [47-50]. The

468 dimeric physiological state of FHR-1 and the other FHRs tested in this study is

469 also likely to enhance their ability, due to increased avidity, to assemble a

470 tripartite complex.

Analysis of the hydrodynamic volume of the Sbi-III-IV:C3d complex using

473 switchSENSE highlighted a significant contraction of the normally extended

474 conformation Sbi-III-IV structure [34] caused by the K173A substitution in

475 domain III (Table 2). SAXS analysis confirms these findings, showing a partially

476 kinked N-terminal structure of domain III in K173A with reduced conformational

477 freedom (Figure 4e-g). The contraction of the Sbi-III-IV structure caused by the

478 K173A substitution suggests that the normally flexible and extended

479 conformation of domain III plays an important role in the recruitment of FHRs,

480 especially FHR-5 into the tripartite complex after the initial interaction between

481 Sbi-IV and C3b. Previous structural analyses of the Sbi's domain III, using NMR,

revealed that this domain is indeed natively unfolded [51]. 
Based on the structural and functional information described here we

485 decided to construct a Sbi-III-IV-Ag85b fusion construct that could be used to

486 test its effect on the immune response against this model antigen in vivo. We

487 chose Mycobacterium tuberculosis Ag85b, a fibronectin-binding protein with

488 mycolyltransferase activity [52], because it is known to be immunogenic and

489 previously suggested as a vaccine candidate [53]. Indeed, there is evidence that

490 Ag85b can elicit both humoral and cellular immune reactions in patients with TB,

491 but there is conflicting evidence of its efficacy as a vaccine [54,55], suggesting

492 adjuvants may improve its overall immunogenicity. This target also gives scope

493 to allow further testing in animal models of disease [56]. Structural analysis,

494 using Circular Dichroism and SAXS confirmed that secondary structural elements

495 of both parent proteins have been preserved in the fusion protein construct and

496 that the crucial functional Sbi domains are accessible for interactions with

497 complement (Figure 5 and Supporting Figure S5). We also show that the

498 Sbi-III-IV-Ag85b fusion construct can induce AP activation and is opsonized with

499 C3 breakdown products (Figure 5c).

500 With AP activation in human and mouse serum confirmed (Figures 5 and 6),

501 we opted to use straightforward immune response, IgG titre, analysis to

502 demonstrate the potential of Sbi-III-IV to trigger complement in vivo and act as a

503 vaccine adjuvant in a mouse model, in a similar manner to many previous studies

504 [57]. Our data herein firstly indicates that Sbi-III-IV can activate mouse 
complement in an analogous manner to that of the human complement system. This obviously allows direct analysis of these pro-vaccine compounds in both

507 mouse and human model systems (a huge advantage to previous C3d based 508 adjuvants) [13], indeed Sbi-III-IV has acted as a C3 activator in all species tested 509 thus far (data not shown). As predicted from the in vitro work, the opsonisation 510 of fusion proteins or co-immunised antigen by mouse complement breakdown

511 fragments results in a significant increase in the immunogenicity of Ag85b, with

512 increased IgG titres noted in the presence of fused or co-immunised Ag85b 513 (Figure 6). The adjuvant function both increased the intensity of the response 514 and the rate of the response when compared to Ag85b immunized alone. We will 515 need to further explore the potency of this response to that of common adjuvants 516 and with a mix of target antigens to fully assess the utility of Sbi-III-IV as a 517 universal vaccine adjuvant. For instance, comparison of the action of Sbi-III-IV to 518 the Glaxo-Smith-Kline's adjuvant systems, particularly AS01 [58], or to MF59 [59] 519 may be of key interest and recent approaches may provide ideal pre-clinical 520 model systems to facilitate this $[60,61]$ before progression to clinical studies. 521 The work is ongoing but the data herein demonstrate the initial proof of concept.

In summary, we have demonstrated that Sbi-III-IV triggers consumption of 524 complement component C3 via activation of the alternative complement 525 pathway, by acting as a competitive antagonist of FH via the recruitment of FHRs 526 into dimeric tripartite complexes that can protect C3b bound to Sbi (Figure 6d). 
527 It is likely this provides a stable nidus for alternative pathway mediated C3

528 convertase generation, i.e. local fluid phase $\mathrm{C} 3 \mathrm{bBb}$ generation that overwhelms

529 any local complement regulators, providing the potential for bystander lysis or

530 opsonisation of surfaces. Our ability to harness this potential, targeting

531 complement opsonisation to the surface of an antigen (in this case from

532 Mycobacterium) and therefore use Sbi-III-IV as a vaccine adjuvant clearly

533 demonstrates Sbi-III-IV has great potential for use with a range of antigens

534 across multiple species, including humans, although more work remains to make

535 that a reality. 


\section{Materials and Methods}

539 Factor $\mathrm{H}(\mathrm{FH}), \mathrm{C} 3 \mathrm{~b}$, factor $\mathrm{B}(\mathrm{FB})$, factor $\mathrm{D}(\mathrm{FD})$, factor I (FI), properdin $(\mathrm{FP})$,

540 FI-depleted serum, goat anti-human C3 polyserum and goat anti-human FB

541 polyserum were purchased from Complement Technologies (Tyler, TX). FHR-1 1 -2,

542 FHR-1, -2 and -5 used in the tripartite complex reconstruction and binding

543 competition assay were produced using Chinese Hamster ovary cell culture (as

544 previously described [62]). Horse radish peroxidase (HRP)-conjugated rabbit

545 anti-goat immunoglobulin polyserum and HRP-conjugated Streptavidin were

546 acquired from Sigma Aldrich. HRP-conjugated goat anti-rabbit immunoglobulin

547 G (Thermo Fisher, catalog no. 815-968-0747), HRP-conjugated rabbit anti-mouse

548 immunoglobulin G (Thermo Fisher, catalog no. 31452) and biotin-conjugated FH

549 monoclonal antibody OX24 (catalog no. MA5-17735) were purchased from

550 Thermo Fisher Scientific. The goat anti-human FH polyclonal serum (catalog no.

$551341276-1 \mathrm{ml}$ ) that was previously used to detect human FH and FHR-1 was

552 purchased from Merck Millipore. Human C3 was purified from mixed pool

553 citrated human plasma (TCS Bioscience, PR100) using polyethylene glycol 4000

554 precipitation, anion and cation exchange chromatography as previously

555 described [63]. A pET15b-C3d construct was acquired from Prof. David E.

556 Isenman and transformed into Escherichia coli (E. coli) stain BL21 (DE3),

557 recombinant C3d was then expressed and purified using a previously described 
558 protocol [64]. Lyophilized normal human serum (NHS) was purchased from Euro

559 Diagnostica (catalog no. PC300). Additional proteins and antibodies are 560 described in the specific experimental sections.

Sbi-III-IV constructs

563 The expression and purification of the N-terminally $6 \times$ His tagged recombinant

564 Sbi-III-IV from a pQE30:sbi-III-IV construct were described previously [24].

Sbi-III-IV mutagenesis

567 Mutations in the Sbi-III-IV sequence were introduced using the QuikChange II XL

568 site-directed mutagenesis kit (Agilent Technologies), the primers used are listed

569 in Supplementary Table S1. The mutated pQE30:sbi-III-IV plasmids were 570 sequenced to confirm the success of the mutagenesis. SDS-PAGE profiles of all 571 the Sbi-III-IV mutant proteins used in this study are shown in Supplementary 572 Figure S1.

575 Lyophilized NHS was re-suspended in chilled $\mathrm{dH}_{2} \mathrm{O}$ to a $2 \times$ concentration. Equal 576 volumes of $2 \times$ NHS and Sbi $(10 \mu \mathrm{M})$ were combined. Sbi treated sera were then 577 incubated in a thermocycler at $37^{\circ} \mathrm{C}$ for $30 \mathrm{~min}$. Treated serum samples were 578 collected at time intervals, $0.5 \mu \mathrm{l}$ of serum was loaded on an SDS-PAGE gel 579 analyzed under reducing condition. The proteins were Western blotted, and the 
bioRxiv preprint doi: https://doi.org/10.1101/413294; this version posted September 10, 2018. The copyright holder for this preprint (which was not certified by peer review) is the author/funder, who has granted bioRxiv a license to display the preprint in perpetuity. It is made available under aCC-BY-NC-ND 4.0 International license.

580 blots were probed with anti-C3d, anti-Sbi, anti-C3a or anti-factor B antibodies.

581

582 A hemolytic assay was modified from a previously published procedure [65] to

583 measure Sbi induced consumption of C3. Briefly, rabbit erythrocytes (TCS

584 Bioscience) were resuspended in GVB buffer (5 mM veronal, $145 \mathrm{mM} \mathrm{NaCl}, 10$

$585 \mu \mathrm{M}$ EDTA, $0.1 \%(\mathrm{w} / \mathrm{v})$ gelatin) by washing three times via centrifugation at 600

$586 g$ for 6 mins. The concentration of rabbit red cells to be used in each experiment

587 was determined by adding a stock of $5 \mu$ l of erythrocytes to $245 \mu$ l of water to

588 give complete lysis and then re-adjusting cell concentration until an optical

589 density reading of $0.7\left(\mathrm{~A}_{405}\right)$ was reached. Lysis experiments were conducted in

590 two steps, first, $15 \mu \mathrm{l}$ of NHS, $5 \mu \mathrm{l}$ of $\mathrm{Mg}^{+}$-EGTA $(70 \mathrm{mM} \mathrm{MgCl} 2$ and $100 \mathrm{mM}$

591 EGTA), $20 \mu$ of protein in E2 buffer was mixed and pre-incubated at $37{ }^{\circ} \mathrm{C}$ for 30

592 mins. Subsequently, $5 \mu \mathrm{l}$ of rabbit erythrocyte was added and incubated for an

593 additional 30 mins at $37^{\circ} \mathrm{C}$. At the end of the incubation, $150 \mu \mathrm{l}$ of quenching

594 buffer (GVB supplemented with $10 \mathrm{mM}$ EDTA) was added. The cells were

595 pelleted by centrifugation at $1500 \mathrm{~g}$ for $10 \mathrm{~min}$, and absorbance $\left(\mathrm{A}_{405}\right)$ of $100 \mu \mathrm{l}$

596 of supernatant measured. Post-consumption lysis percentage was calculated as

$597100 \times\left(\left(A_{405}\right.\right.$ test sample- $A_{405} 0 \%$ control $) /\left(A_{405} 100 \%-A_{405} 0 \%\right.$ control $\left.)\right)$.

598

599 In vitro complement activation using Sbi III/IV-Ag85b in mouse serum

600 Mouse serum was collected from male $\mathrm{Cr} 2 \%$ mice by cardiac puncture and

601 allowed to clot fully on ice for 4 hours followed by separation of serum by 
bioRxiv preprint doi: https://doi.org/10.1101/413294; this version posted September 10, 2018. The copyright holder for this preprint (which

was not certified by peer review) is the author/funder, who has granted bioRxiv a license to display the preprint in perpetuity. It is made available under aCC-BY-NC-ND 4.0 International license.

602 centrifugation at $2000 \mathrm{~g}$ in a refrigerated centrifuge. Serum was then mixed with 603 Sbi III/IV or Sbi III/IV-Ag85b, ensuring that the amount of Sbi III/IV in each 604 preparation was equivalent. The reaction was stopped at $0,30,60$ and 120 605 minutes, by the addition of reducing sample buffer, boiled for $5 \mathrm{~min}$ and analysed 606 on a $10 \%$ SDS-PAGE gel. After transfer to nitrocellulose the blots were probed 607 with Rabbit anti-C3d (1/1000, DAKO, A0063) and Goat anti-Rabbit-HRPO 608 (1/2000, 111-035-046-JIR, Stratech), developed with ECL substrate (Pierce) and 609 exposed to X-Ray film for $2 \mathrm{~min}$.

612 A switchSENSE DRX 2400 instrument (Dynamic Biosensors) was used to 613 characterize the binding kinetics and protein size changes based on switchSENSE 614 technology $[66,67]$. Purified Sbi-III-IV-cys, K173A, R231A and their ligand C3d 615 were sent to Dynamic Biosensor's protein analyzing facility for binding kinetic 616 and hydrodynamic diameter analysis. In the case of a protein binding event, 617 based on the real-time measurements of the switching dynamics in a range of 618 ligand concentrations, binding rate constants ( $k_{\mathrm{ON}}$ and $k_{\mathrm{OFF}}$ ) and dissociation 619 constants $\left(K_{\mathrm{D}}\right)$ can be analysed [67]. Alternatively, under saturated binding 620 conditions, the switching dynamic of the protein (or protein complex) can be 621 compared with the switching dynamics of bare DNA and with a biophysical 622 model with which the size of the immobilized protein (or protein complex) can 623 be determined. For determination of Sbi-III-IV:C3d binding kinetic parameters, 
$624130 \mathrm{nM}, 100 \mathrm{nM}, 70 \mathrm{nM}$ and $40 \mathrm{nM}$ of C3d were applied sequentially onto the

625 Sbi-III-IV immobilized microchip. All Sbi:C3d complexes' hydrodynamic

626 diameters were estimated at a C3d concentration of $130 \mathrm{nM}$.

Fluorometric assay

629 Fluorometric C3b breakdown assay was performed using a black 96 well

630 microplate (Thermofisher, M33089) in a TECAN Spark 20M

631 temperature-controlled fluorescence plate reader. Excitation was at $386 \mathrm{~nm}$ and 632 emission was recorded at $475 \mathrm{~nm}$ with a 20-nm bandwidth. The control C3b 633 breakdown rate, performed in PBS, contained $100 \mu \mathrm{l}$ of $1 \mu \mathrm{M} \mathrm{C} 3 \mathrm{~b}, 160 \mathrm{nM} \mathrm{FH}, 17$ $634 \mathrm{nM}$ of FI and $10 \mu \mathrm{M}$ ANS, and was scanned every $5 s$ for $15 \mathrm{~min}$. To study the 635 interruption of C3b breakdown, $32 \mathrm{nM}$ of FHR was either added alone or in 636 combination with $1 \mu \mathrm{M}$ of Sbi-III-IV. Data were collected at $25^{\circ} \mathrm{C}$, normalized by 637 Excel using the equation "Percentage of $\mathrm{C} 3 \mathrm{~b}=\left(\left(F_{\mathrm{X}}-\left(F_{15 \mathrm{~min}}\right)\right) /\left(F_{15 \mathrm{~min}}-F_{0 \mathrm{~min}}\right)\right) * 100$ " 638 and plotted by Graphpad Prism.

Small angle X-ray scattering

Synchrotron radiation X-ray scattering from solutions of the

642 Sbi-III-IV:C3d:FHR-1 tripartite complex, the Sbi-III-IV(K173A):C3d complex, and 643 the Sbi-III-IV-Ag85b fusion protein were collected at the EMBL P12 beamline of 644 the storage ring PETRA III (DESY, Hamburg, Germany). Images were collected 645 using a photon counting Pilatus-2M detector and a sample to detector distance of 
bioRxiv preprint doi: https://doi.org/10.1101/413294; this version posted September 10, 2018. The copyright holder for this preprint (which was not certified by peer review) is the author/funder, who has granted bioRxiv a license to display the preprint in perpetuity. It is made available under aCC-BY-NC-ND 4.0 International license.

$6463.1 \mathrm{~m}$ and a wavelength $(\lambda)$ of $0.12 \mathrm{~nm}$ covering the range of momentum transfer

647 (s) $0.1<\mathrm{s}<4.5 \mathrm{~nm}^{-1}$; with $\mathrm{s}=4 \pi \sin \theta / \lambda$. Different solute concentrations were

648 measured using a continuous flow cell capillary. To monitor radiation damage,

64920 successive $50 \mathrm{~ms}$ exposures were compared and frames displaying significant

650 alterations were discarded. The data were normalized to intensity of the

651 transmitted beam and radially averaged; the scattering of the buffer was

652 subtracted, and the different curves were scaled for solute concentration. The

653 forward scattering $\mathrm{I}(0)$, the radius of gyration $(\mathrm{Rg})$ along with the probability

654 distribution of the particle $(\mathrm{p}(\mathrm{r}))$ and the maximal dimension $\left(\mathrm{D}_{\max }\right)$ were 655 computed using the automated SAXS data analysis pipeline SASFLOW [68].

656 For the Sbi-III-IV-Ag85b fusion protein data quality was improved with 657 SEC-SAXS mode and the parallel analysis of light scattering data in a similar 658 manner as described in Gräwert et al. [69]. Frames comprising solely the 659 monomeric version of the fusion protein were averaged and used for further 660 processing after background subtraction.

661 The molecular masses (MM) were evaluated by comparison of the forward 662 scattering with that from a reference solution of BSA and based on the Porod 663 volumes of the constructs. With SAXS, the former estimation of MM is within an 664 error of $10 \%$, provided the sample and standard concentrate are determined 665 accurately. DAMMIF was used to compute the ab initio shape models. For this, 10 666 independent models fitting the experimental scattering curves were generated 667 and compared to each other. More detailed modelling was obtained with Coral. 
668 Here, existing partial crystal structure of the Sbi-IV:C3d complex was extended

669 with 60 additional beads placed at the N-terminus of Sbi-IV to mimic the missing

670 Sbi-III domain. Here too, 10 independent runs were performed, and the degree of

671 variation addressed. Further analysis of the flexibility of the samples was

672 addressed with Ensemble Optimization Method (EOM). For this, ensembles of

673 models with variable conformations are selected from a pool of randomly

674 generated models such that the scattering from the ensemble fits the

675 experimental data, and the distributions of the overall parameters (e.g. $D_{\max }$ ) in

676 the selected pool are compared to the original pool.

677 The proteins in the Sbi-III-IV:C3d:FHR-1 tripartite complex were combined

678 1:1:1 at a concentration of $45 \mu \mathrm{M}$. The Sbi-III-IV(K173A):C3d complex were

679 formed at a $1: 1$ ratio at $240 \mu \mathrm{M}(12 \mathrm{mg} / \mathrm{ml})$. PDB structure 2 wy8 (Sbi-IV:C3d

680 complex) was used to model the complex using and compared with SAXS data

681 previously recorded [34]. The Sbi-III-IV-Ag85b fusion protein was provided at 29,

68272 and $145 \mu \mathrm{M}$ concentrations (1.45, 3.6 and $7.2 \mathrm{mg} / \mathrm{ml}$, respectively). The

683 samples were dialysed against PBS, which was also used for background

684 subtraction. From all samples concentration series were measured to exclude

685 any concentration dependent alterations.

686

688 Tripartite complexes were analyzed by surface plasmon resonance (SPR)

689 technology using a Biacore S200 (GE Healthcare). All experiments were 
692 supplemented with $1 \mathrm{mM}$ of $\mathrm{MgCl}_{2}\left(\mathrm{HBST}^{+}\right)$to be compatible with AP

693 amplification condition. On the chip surface $800 \mathrm{RU}$ of C3b was opsonized via AP

694 C3 convertase through a method described before $[70,71]$. The iC3b surface was 695 produced by injecting of repetitive cycles of FH and FI across a C3b opsonized 696 surface, the completeness of the conversion was confirmed by the inability of FB 697 binding. A separate chip surface was made by amine coupling 600 RU of 698 recombinant C3d (CompTech, USA). In all SPR experiments, response differences 699 were derived using the signal from a flow cell to subtract the parallel reading 700 from a reference flow cell that blocked with carbodiimide, $N$-hydroxysuccinimide 701 and ethanolamine. Analytes were injected in duplicate (at $30 \mu \mathrm{l} / \mathrm{min}$ for $200 \mathrm{~s}$ ) 702 followed by running buffer for $300 s$ and a regeneration phase involving injection 703 of regeneration buffer (10 mM sodium acetate, $1 \mathrm{M} \mathrm{NaCl} \mathrm{pH} \mathrm{4.0)} \mathrm{for} 60 \mathrm{~s}$. To 704 analyze Sbi-III-IV binding and the assembly of tripartite complex, concentration 705 series of Sbi-III-IV WT or K173A were flowed cross separately or co-injected 706 with FH, FHR-1, FHR-2, FHR-5 or $\mathrm{FH}_{19-20}$ at a fixed concentration $(100,12.5,20$, 70725 or $20 \mathrm{nM}$ respectively).

709 The C3 convertase DAA assay was performed on a CM5 chip amine coupled with $710500 \mathrm{RU} \mathrm{C} 3 \mathrm{~b}$, using $\mathrm{HBST}^{+}$as running buffer throughout. A mixture of analytes for 711 building C3 convertase were flowed across, including FB and FD in addition to 
712 various FH reagent combinations (FH or FH and FHR-1 or FH, FHR-1 and -5). The

713 various FH reagents combinations were also flow across separately in order to

714 derive the sensorgram for C3 convertase. To examining Sbi bound C3 convertase,

$7152 \mu \mathrm{M}$ of wild-type Sbi-III-IV was added to the mixture of analytes for building C3

716 convertase. The various FH reagent combinations spiked with Sbi were flowed

717 across separately in order to derive the sensorgram for Sbi bound C3 convertase.

718 Each injection cycle includes Injection of the C3 convertase mixture for 200-300

$719 s$, followed by running buffer for 300-400 $s$ and two consecutive $60 s$

720 regeneration phases.

FH/FHR-1 Competition assay

723 C3b was diluted in carbonate buffer ( $\mathrm{pH} 9.5)$ and coated on to wells of a Nunc

724 MaxiSorp plate $(0.25 \mu \mathrm{g} /$ well $)$ for $16 \mathrm{~h}$ at $4^{\circ} \mathrm{C}$. The wells were blocked with PBST

725 (PBS with $0.1 \%$ Tween 20) supplemented with and $2 \%$ BSA for $1 \mathrm{~h}$ at $37^{\circ} \mathrm{C}$, and

726 then washed with PBST buffer. Doubly diluted concentration series (9-600 nM)

727 of FHRs, $\mathrm{FH}_{19-20}$, FHR-11-2 in PBST-2\% BSA were then added to the wells,

728 together with a constant concentration of FH (25 nM) and Sbi-III-IV (1000 nM).

729 The plate was incubated for $1 \mathrm{~h}$ at $37{ }^{\circ} \mathrm{C}$, then washed with PBST. $50 \mu \mathrm{l}$ of

730 monoclonal anti-FH antibody OX-24 (specific to the FH SCR domain 5) diluted

731 with PBS-2\% BSA $(0.6 \mu \mathrm{g} / \mathrm{ml})$ was added to the wells and the plate incubated for

732 a further $1 \mathrm{~h}$. The wells were washed with PBST, and $50 \mu \mathrm{l}$ sheep anti-mouse IgG

733 (1:5000 dilution in PBST-2\% BSA) was added to the wells for $1 \mathrm{~h}$ at $37{ }^{\circ} \mathrm{C}$. The 
734 wells were washed again and the conjugate was detected using TMB ELISA

735 substrate solution, which was added to the wells for $5 \mathrm{~min}$. The colour reaction

736 was stopped by $10 \% \mathrm{H}_{2} \mathrm{SO}_{4}$ and the plate was read at $A_{450}$ using a plate reader.

The DNA sequence coding for Sbi-III-IV ( $s b i_{448-798)}$ was fused to the 5 ' end of the

DNA sequence for Ag85b (ag85b121-975) via a linker region of $84 \mathrm{bp}$

741 (Supplementary Figure S6). The fusion gene was commercially synthesized and

742 ligated into the $\mathrm{pET} 15 \mathrm{~b}$ vector, containing an ampicillin resistance cassette and a

743 T7 promoter. The pET15b:sbi-III-IV-ag85b plasmid was verified using

744 sequencing, and the resulting construct encoded an N-terminally his-tagged

745 Sbi-III-IV-Ag85b protein. E. coli BL21 (DE3) cells harbouring the

746 pET15b:sbi-III-IV-ag85b plasmid were grown in LB broth supplemented with

$747100 \mu \mathrm{g} / \mathrm{ml}$ ampicillin to an $A_{600}=0.4-0.6$. Protein expression was induced with

$7480.5 \mathrm{mM}$ IPTG and by incubating the cells at $17^{\circ} \mathrm{C}$ for $16 \mathrm{~h}$. Bacteria were

749 harvested, lysed using sonication (80\% amplitude, for six $10 \mathrm{~s}$ bursts) in the

750 presence of protease inhibitor cocktail (set VII-Calbiochem, Merck), and the

751 protein initially purified using nickel-affinity chromatography (His-Trap column,

752 GE Healthcare) with a gradient of 0-0.5 M imidazole in $50 \mathrm{mM}$ Tris, $150 \mathrm{mM} \mathrm{NaCl}$,

$753 \mathrm{pH}$ 7.4. It was further purified using size-exclusion chromatography (Hi-Load

754 16/60 Superdex S200 column, GE Healthcare) equilibrated in 20 mM Tris, 150

$755 \mathrm{mM} \mathrm{NaCl}, \mathrm{pH}$ 7.4. Fractions containing protein were pooled and concentrated. 
bioRxiv preprint doi: https://doi.org/10.1101/413294; this version posted September 10, 2018. The copyright holder for this preprint (which was not certified by peer review) is the author/funder, who has granted bioRxiv a license to display the preprint in perpetuity. It is made available under aCC-BY-NC-ND 4.0 International license.

756 Protein concentration was measured at $A_{280}$.

757

758

Analysis of Sbi-III-IV-Ag85b fusion protein AP complement activity

759

Alternative pathway (AP) activity of Sbi III-IV-Ag85b-treated NHS samples was

760

analysed using the ELISA-based WIESLAB ${ }^{\circledR}$ (Euro Diagnostica) complement

761

system AP assay. Sbi-III-IV-Ag85b was mixed with normal human serum (NHS)

762 at a $1: 1$ volume ratio and incubated for $30 \mathrm{~min}$ at $37^{\circ} \mathrm{C}$ in a thermal cycler.

763 Treated serum was then diluted with AP diluent (blocking the activation of the

764 other two complement pathways) by 1 in 20 . From this point the manufacturer's

765 instructions were followed. A blank (AP diluent), positive control (NHS) and

766 negative control (heat-inactivated NHS) were also recorded. Complement

767 activation was converted to residual AP activity (\%) using the equation: (sample

768 - negative control)/(positive control - negative control) x 100.

769

770

C3 fragment deposition on Sbi-III-IV-Ag85b fusion protein

771 The method used is similar to that described for WT Sbi-III-IV. Lyophilised NHS

772 (Euro Diagnostica) was re-suspended in chilled $\mathrm{dH}_{2} \mathrm{O}$. Sbi-III-IV-Ag85b (100 $\left.\mu \mathrm{M}\right)$

773 was mixed with NHS in a $1: 1$ ratio, and incubated for $1 \mathrm{~h}$ at $37{ }^{\circ} \mathrm{C}$ in a

774 thermocycler. Samples were taken at regular intervals $(0,5,15,30,60 \mathrm{~min})$, and

775 separated by SDS-PAGE followed by Western blot analysis using either rabbit

776 anti-Sbi (1.5: 5000 dilution), rabbit anti-C3d (1.5:5000 dilution) or mouse

777 anti-Ag85b (1:1000 dilution) polyclonal antibodies and detected using 
bioRxiv preprint doi: https://doi.org/10.1101/413294; this version posted September 10, 2018. The copyright holder for this preprint (which was not certified by peer review) is the author/funder, who has granted bioRxiv a license to display the preprint in perpetuity. It is made available under aCC-BY-NC-ND 4.0 International license.

778 HRP-conjugated secondary antibodies (1:2500 goat anti rabbit or 1:1000 goat 779 anti mouse). NHS-only was used as a negative control.

780

\section{Measurement of immune response to Sbi-III-IV-Ag85b fusion protein}

782 Eight week old male mice (wild-type $\mathrm{C} 57 \mathrm{bl} / 6, \mathrm{C} 3 \%$ and $\mathrm{Cr} 2 \%$ ) were bled by tail 783 vein venesection at day -2 . Mice were then immunized at day 0 with molar

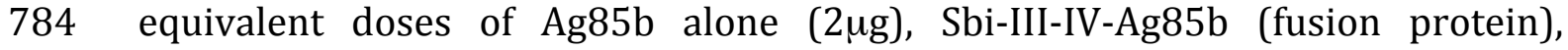
785 Sbi-III-IV alone or a mixture of Sbi-III-IV and Ag85b, as appropriate. Mice were 786 then bled weekly thereafter and plasma stored at $-80^{\circ} \mathrm{C}$ until required for batch 787 analysis. Mice were boosted at day 28 and sacrificed at day 42 .

788 For analysis of IgG response to Ag85b by ELISA, 96 well plates (NUNC maxisorb) 789 were coated with $1 \mu \mathrm{g} / \mathrm{ml}$ Ag85b (Abcam, UK) or $1.35 \mu \mathrm{g} / \mathrm{ml}$ Sbi III/IV-Ag85b in 790 carbonate buffer at $50 \mu \mathrm{l}$ per well and incubated at $4^{\circ} \mathrm{C}$ for $16 \mathrm{~h}$. Plates were 791 washed with $0.01 \%$ PBS-Tween and a 1\% BSA blocking solution was applied for $7921 \mathrm{~h}$ at $20^{\circ} \mathrm{C}$. Serum samples were diluted to $1 / 50$ or $1 / 100$ in $0.01 \%$ PBS-Tween, 793 added at $50 \mu \mathrm{l}$ per well and incubated for $1 \mathrm{~h}$ at $20^{\circ} \mathrm{C}$. Plates were washed and 794 secondary antibody (sheep anti mouse IgG-HRPO, 515-035-071-JIR, Stratech, UK) 795 was added at $1 / 100$ dilution, $50 \mu \mathrm{l}$ per well and incubated for $1 \mathrm{~h}$ at $20^{\circ} \mathrm{C}$. TMB 796 substrate (50 $\mu \mathrm{l}$ per well) was added and allowed to develop for $6 \mathrm{~min}$. The 797 reaction was stopped by the addition of $100 \mu \mathrm{l} 10 \% \mathrm{H}_{2} \mathrm{SO}_{4}$ per well and plates 798 were read at $A_{450}$. A mouse monoclonal anti-Ag85b (Abcam, ab43019) used as a 799 positive control. The mean absorbance \pm SEM of each mouse group is shown. 
bioRxiv preprint doi: https://doi.org/10.1101/413294; this version posted September 10, 2018. The copyright holder for this preprint (which was not certified by peer review) is the author/funder, who has granted bioRxiv a license to display the preprint in perpetuity. It is made available under aCC-BY-NC-ND 4.0 International license.

800 Data for each mouse, at time 0 , has been normalized to the day 0 average

801 reactivity to Ag85b in all mice screened. 


\section{Acknowledgements}

803 This research was funded by the Biotechnology and Biological Sciences Research

804 Council (BBSRC Follow On Fund BB/N022165/1, awarded to JvdE and KJM).

805 AAW was supported by a PhD scholarship granted Raoul and Catherine Hughes

806 and the University of Bath Alumni. KJM, HD and RW were also supported by the

807 MRC and Newcastle University's Confidence in Concept funding. AS thanks

808 the Royal Society URF and Alumni Fund at the University of Bath for funding.

809 MAG was supported by the EMBL interdisciplinary Postdoc Programme under

810 Marie Curie COFUND Actions as well as the Horizon 2020 programme of the

811 European Union, iNEXT (H2020 Grant \# 653706).

812

\section{Author Contributions}

814 JvdE, AGW and KJM conceived the idea of the project. YY, KJM and JvdE designed

815 the experiments. YY, CRB, AAW, RK and JP performed and analysed the

816 experiments. KW and WK conducted and analysed the switchSENSE experiments.

817 MAG and DIS conducted SAXS experiments and oversaw the structural analysis.

818 RK, JP and KJM conducted the in vivo experiments. AS and AW significantly

819 contributed to the discussions about the overall project. YY, CRB, KJM and JvdE

820 wrote and edited the manuscript, with significant contributions from AAW, MAG

821 and DIS.

\section{Competing Financial Interests}


bioRxiv preprint doi: https://doi.org/10.1101/413294; this version posted September 10, 2018. The copyright holder for this preprint (which

was not certified by peer review) is the author/funder, who has granted bioRxiv a license to display the preprint in perpetuity. It is made available under aCC-BY-NC-ND 4.0 International license.

824 KW and WK are employed by Dynamic Biosensors GmbH

$825 \mathrm{KJM}$ is a member of the scientific advisory board of Gemini Therapeutics, Inc,

826 Cambridge, Massachusetts, USA.

827

828 Data availability

829 All data generated or analysed during this study are included in this published

830 article (and its supplementary information files).

831 


\section{References}

833 1. Green, T.D., et al., C3d enhancement of neutralizing antibodies to measles hemagglutinin. Vaccine, 2001. 20(1-2): p. 242-8.

3. Dempsey, P.W., et

4. $\quad$ Fang, Y., et al., Expression of complement receptors 1 and 2 on follicular dendritic cells is necessary for the generation of a strong antigen-specific IgG response. J Immunol, 1998. 160(11): p. 5273-9.

5. Carroll, M.C. and D.E. Isenman, Regulation of humoral immunity by complement. Immunity, 2012. 37(2): p. 199-207.

6. Roozendaal, R. and M.C. Carroll, Complement receptors CD21 and CD35 in humoral immunity. Immunol Rev, 2007. 219: p. 157-66.

7. Popi, A.F., I.M. Longo-Maugeri, and M. Mariano, An Overview of B-1 Cells as Antigen-Presenting Cells. Frontiers in immunology, 2016. 7: p. 138.

8. Chan, O.T., M.P. Madaio, and M.J. Shlomchik, $B$ cells are required for lupus nephritis in the polygenic, Fas-intact MRL model of systemic autoimmunity. J Immunol, 1999. 163(7): p. 3592-6.

855

11. De Groot, A.S., et al., C3d adjuvant effects are mediated through the

12. Carter, R.H. and D.T. Fearon, Polymeric C3dg primes human B lymphocytes

9. Ron, Y., et al., Defective induction of antigen-reactive proliferating $T$ cells in

10. Ron, Y., et al., Defective induction of antigen-reactive proliferating $T$ cells in $B$ cell-deprived mice. II. Anti-mu treatment affects the initiation and recruitment of T cells. Eur J Immunol, 1983. 13(2): p. 167-71. activation of C3d-specific autoreactive T cells. Immunol Cell Biol, 2015. 93(2): p. 189-97.

874 17. Forneris, F., et al., Structures of C3b in complex with factors $B$ and $D$ give 
insight into complement convertase formation. Science, 2010. 330(6012): p. 1816-20.

18. Wu, J., et al., Structure of complement fragment C3b-factor $H$ and implications for host protection by complement regulators. Nat Immunol, 2009. 10(7): p. 728-33.

19. Morgan, H.P., et al., Structural basis for engagement by complement factor H of C3b on a self surface. Nat Struct Mol Biol, 2011. 18(4): p. 463-70.

20. Kajander, T., et al., Dual interaction of factor $H$ with C3d and glycosaminoglycans in host-nonhost discrimination by complement. Proc Natl Acad Sci U S A, 2011. 108(7): p. 2897-902.

21. Goicoechea de Jorge, E., et al., Dimerization of complement factor H-related proteins modulates complement activation in vivo. Proceedings of the National Academy of Sciences, 2013. 110(12): p. 4685-4690.

22. Jozsi, M., et al., Factor H-related proteins determine complement-activating surfaces. Trends Immunol, 2015. 36(6): p. 374-84.

23. Xue, X., et al., Regulator-dependent mechanisms of C3b processing by factor I allow differentiation of immune responses. Nat Struct Mol Biol, 2017. 24(8): p. 643-651.

24. Burman, J.D., et al., Interaction of human complement with Sbi, a staphylococcal immunoglobulin-binding protein indications of a novel mechanism of complement evasion by Staphylococcus aureus. Journal of Biological Chemistry, 2008. 283(25): p. 17579-17593.

25. Smith, E.J., et al., The immune evasion protein Sbi of Staphylococcus aureus occurs both extracellularly and anchored to the cell envelope by binding lipoteichoic acid. Molecular Microbiology, 2012. 83(4): p. 789-804.

26. Haupt, K., et al., The Staphylococcus aureus Protein Sbi Acts as a Complement Inhibitor and Forms a Tripartite Complex with Host Complement Factor H and C3b. Plos Pathogens, 2008. 4(12 ).

27. Csincsi, A.I., et al., Factor H-related protein 5 interacts with pentraxin 3 and the extracellular matrix and modulates complement activation. J Immunol, 2015. 194(10): p. 4963-73.

28. van Beek, A.E., et al., Factor H-Related (FHR)-1 and FHR-2 Form Homo- and Heterodimers, while FHR-5 Circulates Only As Homodimer in Human Plasma. Front Immunol, 2017. 8: p. 1328.

29. Isenman, D.E., et al., Nucleophilic modification of human complement protein C3: correlation of conformational changes with acquisition of C3b-like functional properties. Biochemistry, 1981. 20(15): p. 4458-67.

30. Pangburn, M.K. and H.J. Muller-Eberhard, Kinetic and thermodynamic analysis of the control of C3b by the complement regulatory proteins factors $H$ and I. Biochemistry, 1983. 22(1): p. 178-85.

31. McRae, J.L., et al., Human factor H-related protein 5 has cofactor activity, inhibits C3 convertase activity, binds heparin and C-reactive protein, and associates with lipoprotein. Journal of immunology (Baltimore, Md : 1950), 2005. 174(10): p. 6250-6. 
919

920

921

922

923

924

925

926

927

928

929

930

931

932

933

934

935

936

937

938

939

940

941

942

943

944

945

946

947

948

949

950

951

952

953

954

955

956

957

958

959

960

961

962

32. Tria, G., et al., Advanced ensemble modelling of flexible macromolecules using X-ray solution scattering. IUCr], 2015. 2(Pt 2): p. 207-17.

33. Morgan, H.P., et al., Structural analysis of the C-terminal region (modules 18-20) of complement regulator factor $H$ (FH). PLoS One, 2012. 7(2): p. e32187.

34. Clark, E.A., et al., A structural basis for Staphylococcal complement subversion: X-ray structure of the complement-binding domain of Staphylococcus aureus protein Sbi in complex with ligand C3d. Molecular Immunology, 2011. 48(4): p. 452-462.

35. Franke, D. and D.I. Svergun, DAMMIF, a program for rapid ab-initio shape determination in small-angle scattering. Journal of Applied Crystallography, 2009. 42: p. 342-346.

36. Tuukkanen, A.T., G.J. Kleywegt, and D.I. Svergun, Resolution of ab initio shapes determined from small-angle scattering. IUCrJ, 2016. 3(Pt 6): p. 440-447.

37. Petoukhov, M.V., et al., New developments in the ATSAS program package for small-angle scattering data analysis. Journal of Applied Crystallography, 2012. 45: p. 342-350.

38. Pepys, M.B., Role of complement in induction of antibody production in vivo. Effect of cobra factor and other C3-reactive agents on thymus-dependent and thymus-independent antibody responses. J Exp Med, 1974. 140(1): p. 126-45.

39. Fearon, D.T. and R.H. Carter, The CD19/CR2/TAPA-1 complex of B lymphocytes: linking natural to acquired immunity. Annu Rev Immunol, 1995. 13: p. 127-49.

40. Wessels, M.R., et al., Studies of group B streptococcal infection in mice deficient in complement component C3 or C4 demonstrate an essential role for complement in both innate and acquired immunity. Proc Natl Acad Sci U S A, 1995. 92(25): p. 11490-4.

41. Ahearn, J.M., et al., Disruption of the Cr2 locus results in a reduction in B-1a cells and in an impaired $B$ cell response to T-dependent antigen. Immunity, 1996. 4(3): p. 251-62.

42. Lee, Y., et al., Complement component C3d-antigen complexes can either augment or inhibit $B$ lymphocyte activation and humoral immunity in mice depending on the degree of CD21/CD19 complex engagement. J Immunol, 2005. 175(12): p. 8011-23.

43. Suradhat, S., et al., Fusion of C3d molecule with bovine rotavirus VP7 or bovine herpesvirus type 1 glycoprotein $D$ inhibits immune responses following DNA immunization. Vet Immunol Immunopathol, 2001. 83(1-2): p. 79-92.

44. Fries, L.F., et al., C3b covalently bound to IgG demonstrates a reduced rate of inactivation by factors $H$ and I. Journal of Experimental Medicine, 1984. 160(6): p. 1640-1655.

45. Lutz, H.U. and E. Jelezarova, Complement amplification revisited. Molecular 
963

964

965

966

967

968

969

970

971

972

973

974

975

976

977

978

979

980

981

982

983

984

985

986

987

988

989

990

991

992

993

994

995

996

997

998

999

1000

1001

1002

1003

1004

1005

1006

Immunology, 2006. 43(1-2): p. 2-12.

46. Jozsi, M., Factor $H$ Family Proteins in Complement Evasion of Microorganisms. Front Immunol, 2017. 8: p. 571.

47. Aslam, M. and S.J. Perkins, Folded-back solution structure of monomeric factor $H$ of human complement by synchrotron $X$-ray and neutron scattering, analytical ultracentrifugation and constrained molecular modelling. J Mol Biol, 2001. 309(5): p. 1117-38.

48. Oppermann, M., et al., The C-terminus of complement regulator Factor $H$ mediates target recognition: evidence for a compact conformation of the native protein. Clin Exp Immunol, 2006. 144(2): p. 342-52.

49. Okemefuna, A.I., et al., The regulatory SCR-1/5 and cell surface-binding SCR-16/20 fragments of factor $H$ reveal partially folded-back solution structures and different self-associative properties. J Mol Biol, 2008. 375(1): p. 80-101.

50. Makou, E., A.P. Herbert, and P.N. Barlow, Functional anatomy of complement factor $H$. Biochemistry, 2013. 52(23): p. 3949-62.

51. Upadhyay, A., et al., Structure-Function Analysis of the C3 Binding Region of Staphylococcus aureus Immune Subversion Protein Sbi. Journal of Biological Chemistry 2008. 283(32): p. 22113-22120.

52. Belisle, J.T., et al., Role of the major antigen of Mycobacterium tuberculosis in cell wall biogenesis. Science, 1997. 276(5317): p. 1420-2.

53. Palma, C., et al., The Ag85B protein of Mycobacterium tuberculosis may turn a protective immune response induced by Ag85B-DNA vaccine into a potent but non-protective Th1 immune response in mice. Cell Microbiol, 2007. 9(6): p. 1455-65.

54. Weinrich Olsen, A., et al., Protection of mice with a tuberculosis subunit vaccine based on a fusion protein of antigen $85 \mathrm{~b}$ and esat-6. Infect Immun, 2001. 69(5): p. 2773-8.

55. Olsen, A.W., et al., Protective effect of a tuberculosis subunit vaccine based on a fusion of antigen $85 B$ and ESAT-6 in the aerosol guinea pig model. Infect Immun, 2004. 72(10): p. 6148-50.

56. Horwitz, M.A., et al., Protective immunity against tuberculosis induced by vaccination with major extracellular proteins of Mycobacterium tuberculosis. Proc Natl Acad Sci U S A, 1995. 92(5): p. 1530-4.

57. Toapanta, F.R. and T.M. Ross, Complement-mediated activation of the adaptive immune responses: role of $\mathrm{C} 3 \mathrm{~d}$ in linking the innate and adaptive immunity. Immunol Res, 2006. 36(1-3): p. 197-210.

58. Montoya, J., et al., A randomized, controlled dose-finding Phase II study of the M72/AS01 candidate tuberculosis vaccine in healthy PPD-positive adults. J Clin Immunol, 2013. 33(8): p. 1360-75.

59. Seo, Y.B., et al., Comparison of the immunogenicity and safety of the conventional subunit, MF59-adjuvanted, and intradermal influenza vaccines in the elderly. Clin Vaccine Immunol, 2014. 21(7): p. 989-96.

60. Kirkling, M.E., et al., Notch Signaling Facilitates InVitro Generation of 
Cross-Presenting Classical Dendritic Cells. Cell reports, 2018. 23(12): p. 3658-3672.e6.

61. Cytlak, U., et al., Ikaros family zinc finger 1 regulates dendritic cell development and function in humans. Nature communications, 2018. 9(1): p. 1239.

62. Nichols, E.M., et al., An extended mini-complement factor $H$ molecule ameliorates experimental C3 glomerulopathy. Kidney International, 2015. 88(6): p. 1314-1322.

63. Alsenz, J., et al., Phylogeny of the third component of complement, C3: analysis of the conservation of human CR1, CR2, $H$, and B binding sites, concanavalin A binding sites, and thiolester bond in the C3 from different species. Developmental and Comparative Immunology, 1992. 16(1): p. 63-76.

64. Nagar, B., et al., X-ray crystal structure of C3d: a C3 fragment and ligand for complement receptor 2. Science, 1998. 280(5367): p. 1277-1281.

65. Kerr, $\mathrm{H}_{\text {., }}$ et al., Disease-linked mutations in factor $\mathrm{H}$ reveal pivotal role of cofactor activity in self-surface-selective regulation of complement activation. The Journal of biological chemistry, 2017. 292(32): p. 13345-13360.

66. Langer, A., et al., Protein analysis by time-resolved measurements with an electro-switchable DNA chip. Nature Communications, 2013. 4: p. 1-8.

67. Knezevic, J., et al., Quantitation of Affinity, Avidity, and Binding Kinetics of Protein Analytes with a Dynamically Switchable Biosurface. Journal of the American Chemical Society, 2012. 134(37): p. 15225-15228.

68. Franke, D., A.G. Kikhney, and D.I. Svergun, Automated acquisition and analysis of small angle X-ray scattering data. Nuclear Instruments \& Methods in Physics Research Section a-Accelerators Spectrometers Detectors and Associated Equipment, 2012. 689: p. 52-59.

69. Graewert, M.A., et al., Automated Pipeline for Purification, Biophysical and $X$-Ray Analysis of Biomacromolecular Solutions. Scientific Reports, 2015. 5.

70. Yang, Y., et al., An Engineered Complement Factor $H$ Construct for Treatment of C3 Glomerulopathy. Journal of the American Society of Nephrology : JASN, 2018. 29(6): p. 1649-1661.

71. Harris, C.L., et al., Molecular dissection of interactions between components of the alternative pathway of complement and decay accelerating factor (CD55). The Journal of biological chemistry, 2005. 280(4): p. 2569-78. 


\section{Figure Legends}

1046

1047 Figure 1. Sbi-III-IV induces C3 futile consumption via the alternative complement pathway and thereby causes C3b adduct formation and C3a anaphylatoxin production.

(a) C3 activation and C3b deposition in NHS after incubation with $10 \mu \mathrm{M}$

1051 Sbi-III-IV, visualized using anti-C3d western blot analysis. C3b adducts formed 1052 with serum proteins are indicated. Positions of $\alpha-120$ (C3) $\alpha^{\prime} 110$ (C3b) and $1053 \alpha^{\prime}-68$ (iC3b) are indicated. The 10 minute lag-time in C3 activation we observe 1054 in the presence of excess Sbi-III-IV $(10 \mu \mathrm{M})$ correlates with the delay reported 1055 in the natural C3 "tick-over" process, required for supplying the critical 1056 enzymatic component for the initial fluid phase Alternative Pathway (AP) C3

1057 convertase. (b) Sbi-C3b adduct formation, visualized with anti-Sbi western blot.

1058 These adducts migrate at higher than expected molecular weights (Sbi- $\alpha{ }^{\prime}$ 110: $1059 \sim 160 \mathrm{kDa}$ and Sbi- $\alpha>$ 68: $\sim 120 \mathrm{kDa}$, with expected molecular weights of 125 1060 and $83 \mathrm{kDa}$, respectively) which is caused by the high pI of the Sbi-III-IV 1061 construct $(\mathrm{pI}=9.3)$. Sbi-III-IV has a molecular weight of $14.8 \mathrm{kDa}$, but migrates to $\sim 22 \mathrm{kDa}$ in SDS-polyacrylamide gel due to the positively charged electrophoresis

1063 buffer. (c) C3a anaphylatoxin production, followed using anti-C3a western blot 1064 analysis (showing only the low molecular weight region). (d) FB cleavage, 1065 monitored by anti-FB western blot analysis. (e) Concentration dependent 1066 Sbi-III-IV induced C3 consumption, studied by a rabbit erythrocytes haemolytic 
assay. Rabbit erythrocytes were exposed to normal human serum pre-incubated with Sbi-III-IV (incubated, closed circles) and normal human serum with Sbi-III-IV added at the start of the experiment (not incubated, open circles). (f)

1070 Schematic representation of the relative positions of point mutations that display 1071 the most profound functional defects, K173A and R231A. (g) C3 consumption 1072 profiles of Sbi-III-IV mutants K173A and R231A. For b-e and $h$, one 1073 representative blot of three independent experiments was shown. For (e), four 1074 independent measurements of two experiments were shown. The mean and SD 1075 for each measurement were calculated for all datasets. Curves were fitted using non-linear variable slope (four parameters) function in GraphPad Prism.

Figure 2 Surface plasmon resonance analyses of tripartite complexes. A

1081 Sbi-III-IV were co-injected with plasma purified FH, recombinant FHR-1, FHR-2,

1082 FHR-5 or $\mathrm{FH}_{19-20}$. The red response curves were indicative of binding experiment 1083 in the absence of Sbi. The co-injection experiments of a fixed analyte 1084 concentration in combination with increasing Sbi concentration were depicted 1085 by increasingly dark lines. (c) Relative changes of Sbi-III-IV mediated FH (or FHR) 1086 binding to C3b. By subtracting the co-injection sensorgram (i.e. Sbi+FH) with the 1087 corresponding Sbi binding dataset (Supplementary Figure S3), the changes in FH 1088 (or FHRs) binding was deduced (Supplementary Figure S3). Changes in FH (or 
1089 FHRs) binding were expressed as the relative change, derived from dividing the 1090 Sbi mediated binding by the FH (or FHR) only control, using the 1091 response-difference values at the equilibrated binding point (173.5 s). Each 1092 sensorgram is representative of two experiments. Relative change curves were 1093 fitted using non-linear variable slope (four parameters) function in GraphPad 1094 Prism.

Figure 3 Functional characterization of tripartite complexes in

complement AP regulation. NHS was incubated with Sbi-III-IV, in combination

1099 with specified reagents or just buffer, the consumption of AP activity was

1100 indicated by the protection of rabbit red blood cell from lysis. (a) Pre-incubation

1101 of recombinant FHR-1 or -2 with the presence or absence of Sbi-III-IV. (b)

1102 Pre-incubation of recombinant FHR-5 with the presence or absence of Sbi-III-IV.

1103 (c) Pre-incubation of recombinant $\mathrm{FH}_{19-20}$ or FHR-1 $1_{1-2}$ with the presence or 1104 absence of Sbi-III-IV. Using an ELISA assay, the ability of FHR-1, -2, -5, FH $19-20$ or 1105 FHR-1 1 -2 to modulate FH binding to a C3b coated surface was studied in the 1106 presence of WT (d), no (e) or K173A Sbi-III-IV (f). C3 convertase formation in the 1107 absence (g) or presence of Sbi-III-IV (h) was assessed by flowing factor B (500 $1108 \mathrm{nM})$ and factor D (100 nM) in the presence of FH (2000 nM) or FH +FHR-1 (2000 1109 and $200 \mathrm{nM})$ or FH+FHR-1\&-5 (2000, 200 and $20 \mathrm{nM}$ ) across a surface amine 1110 coupled with 500 RU C3b. To form Sbi bound C3 convertase, experiments were 
1111 conducted in addition of $2000 \mathrm{nM}$ of Sbi-III-IV. Detailed experimental and data

1112 processing procedures are provided in material and methods and Supplementary

1113 Figure S3E. (i) Percentage of intact C3b derived from continuous recording of

1114 ANS fluorescence changes between 465-475 nm spectrum. Baseline C3b

1115 breakdown curve (-) was recorded in the presence of FH and FI, interference

1116 caused by the addition of FHR-5 (+) or FHR-5 in combination of Sbi (++) was also

1117 examined. The data for FHR-1 and FHR-2 are presented in Supplementary Figure

1118 3F. Normalized data was depicted in solid lines, simulated breakdown curves

1119 were shown as dotted-lines. Each curve represents the mean value of three

1120 independent experiments. For (a-f), the mean and standard deviation for each

1121 measurement was calculated; For (g-h), each sensorgram is representative of

1122 two experiments. For (i), simulated breakdown curves were fitted using one

1123 phase exponential decay function in GraphPad Prism.

1125 Figure 4. Structural analysis of the Sbi-III-IV:C3d:FHR-1 tripartite complex.

1126 SAXS solution structure analysis and EOM modeling of the Sbi-III-IV:C3d:FHR-1

1127 tripartite complex: (a) Left panel, fit of the selected ensemble of conformers to

1128 the experimental scattering. Radius of gyration ( $R g$, middle panel), particle

1129 maximum dimension (Dmax, right panel), and distribution histograms of the

1130 selected conformers versus the pool. (b) Kratky plot of the tripartite complex. c)

1131 Examples of rigid body models of the selected conformers corresponding to the

1132 histogram peaks. The volume fraction of each species is indicated. The relative 
1133 positions of C3d, Sbi-III-IV and FHR-1 in the dimeric tripartite complex are

1134 indicated, with C3d in red, Sbi-IV in dark blue, Sbi-III in turquoise and FHR-1 in

1135 orange. (d) Schematic representation of the dimeric Sbi-III-IV:C3d:FHR-1

1136 tripartite complex.

1137 (e) Comparison of the solutions structure of wild-type Sbi-III-IV:C3d and 1138 mutated version Sbi-III-IV(K173A):C3d of the dual complex. Radius of gyration

$1139(R g)$, particle maximum dimension (Dmax), and distribution histograms of the 1140 selected conformers versus the pool are shown in Supplementary Figure S4A. (f)

1141 Ab initio shape reconstruction shown as gray spheres in comparison to the 1142 partial crystal structure Sbi-IV:C3d (2wy8). (g) Examples of rigid body models.

1143 Complete set of models as well as flexibility assessment is presented in 1144 supplement Figures S5. C3d in shown red, Sbi-IV in dark blue, and Sbi-III in 1145 turquoise.

1147 Figure 5. Structural and functional analysis of the Sbi-III-IV-Ag85b fusion

1148 protein. (a) Schematic structure of the Sbi-III-IV-Ag85b fusion protein (see fo 1149 details Supplementary Figure S9). (b) SAXS analysis of the fusion protein 1150 indicates a monomeric molecule with a radius of gyration of $\mathrm{R}_{\mathrm{g}}=3.7 \mathrm{~nm}$ and 1151 maximum particle size of $D_{\max }=15 \mathrm{~nm}$. The various molecular mass estimation 1152 range from $44-51 \mathrm{kDa}$ and are comparable with a predicted monomer mass of $115350 \mathrm{kDa}$. The 10 independent ab initio models obtained with DAMMIF are similar 1154 to each other, and according to the $\chi^{2}$ values that estimate the goodness of the fit, 
1155 the final structures fit well with the experiment. More detailed modelling with

1156 Coral and EOM show that the flexibility of the missing structural information is

1157 restricted. (c) C3 activation and C3-fragment deposition in NHS after incubation

1158 with Sbi-III-IV-Ag85b $(100 \mu \mathrm{M})$ or Ag85b $(100 \mu \mathrm{M})$, visualized using anti-Sbi and

1159 anti-C3d western blot analysis. Resultant higher molecular weight bands with

1160 Sbi III-IV-Ag85b were identified as Sbi-III-IV-Ag85b with two covalently attached

1161 C3b $\alpha^{\prime}$ chains; Sbi-III-IV-Ag85b with two iC3b $\alpha^{\prime}-68$ chains and Sbi-III-IV-Ag85b

1162 with two C3d molecules. Ag85b alone is unable to activate C3 as indicated by the 1163 presence of an intact C3 $\alpha$-chain.

1165 Figure 6. Sbi-III-IV is an effective adjuvant in mice.

1166 (a) Freshly prepared CD21\% mouse serum was mixed with Sbi-III-IV-Ag85b or 1167 just Sbi-III-IV. The reaction was stopped at various time points $(0,30,60,120$

1168 minutes). Western blot was developed with rabbit anti-C3 at 1/1000 and goat 1169 anti-rabbit at $1 / 2000$. C3d is shown as confirmation that C3 has been activated 1170 and broken down. (N) is $\mathrm{Cr} 2 \%$ serum incubated for 120 minutes with saline. (b) $1171 \mathrm{C} 57 \mathrm{Bl} / 6$ mice (groups of 6) where immunised intraperitoneally with either $11722.7 \mu \mathrm{g}$ Sbi-III-IV-Ag85b protein, $2 \mu \mathrm{g}$ Ag85b, or 0.7 $\mu \mathrm{g}$ Sbi-III-IV plus $2 \mu \mathrm{g}$ Ag85b in $1173150 \mathrm{mM} \mathrm{NaCl}$ solution, followed by weekly bleed and boosted (day 28) before 1174 terminal bleed at day 49. Serum IgG reactivity to Ag85b was measured over time 1175 by ELISA. Sera was diluted $1 / 50$ and the mean absorbance \pm SEM of each mouse 1176 group is shown. All data has been normalised to the day 0 average of all WT mice. 
1177 (c) The previous experiment was repeated in $\mathrm{C} 57 \mathrm{Bl} / 6$ mice deficient of $\mathrm{C} 3$

$1178\left(\mathrm{C}^{-/}\right)$and complement receptor type I and $2\left(\mathrm{Cr}^{\%} \%\right)$. Data is representative of at

1179 least 2 repeats. (d) Schematic representation of the dimeric Sbi-III-IV:C3d:FHR-1

1180 solution structure providing a nidus for AP C3 convertase generation that

1181 overwhelms local complement regulators, leading to the opsonisation of the

1182 nearby antigen surface by C3 break-down products that help facilitate the

1183 co-ligation of the B cell antigen receptor (BCR) with complement receptor 2 (CR2)

1184 thereby lowering the threshold for B cell activation. 

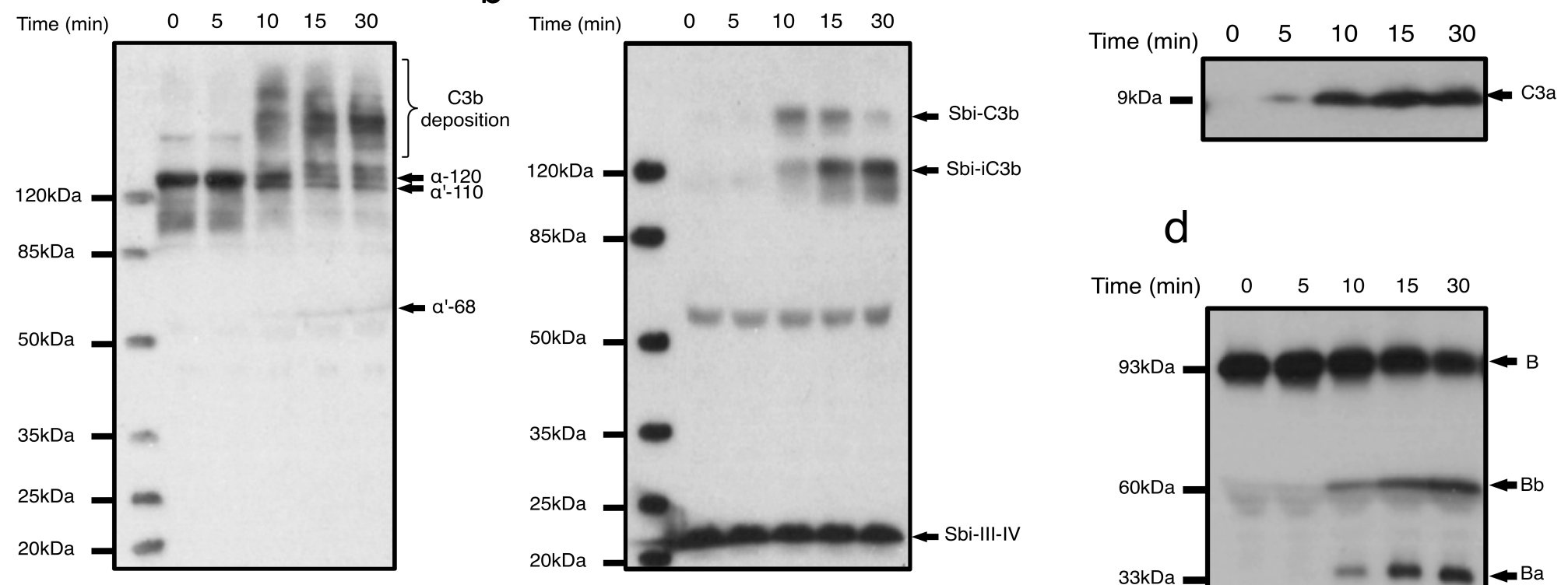

d

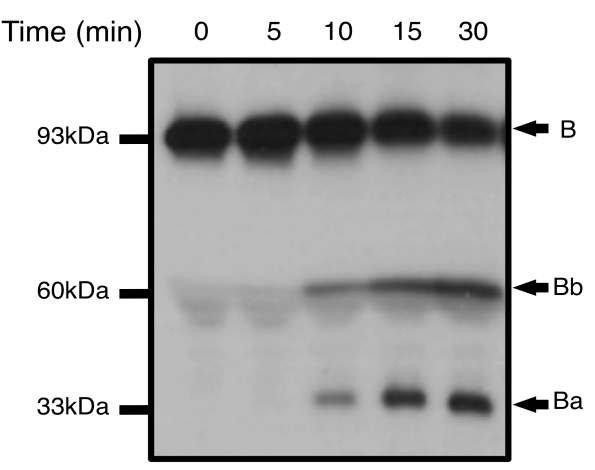

e

f
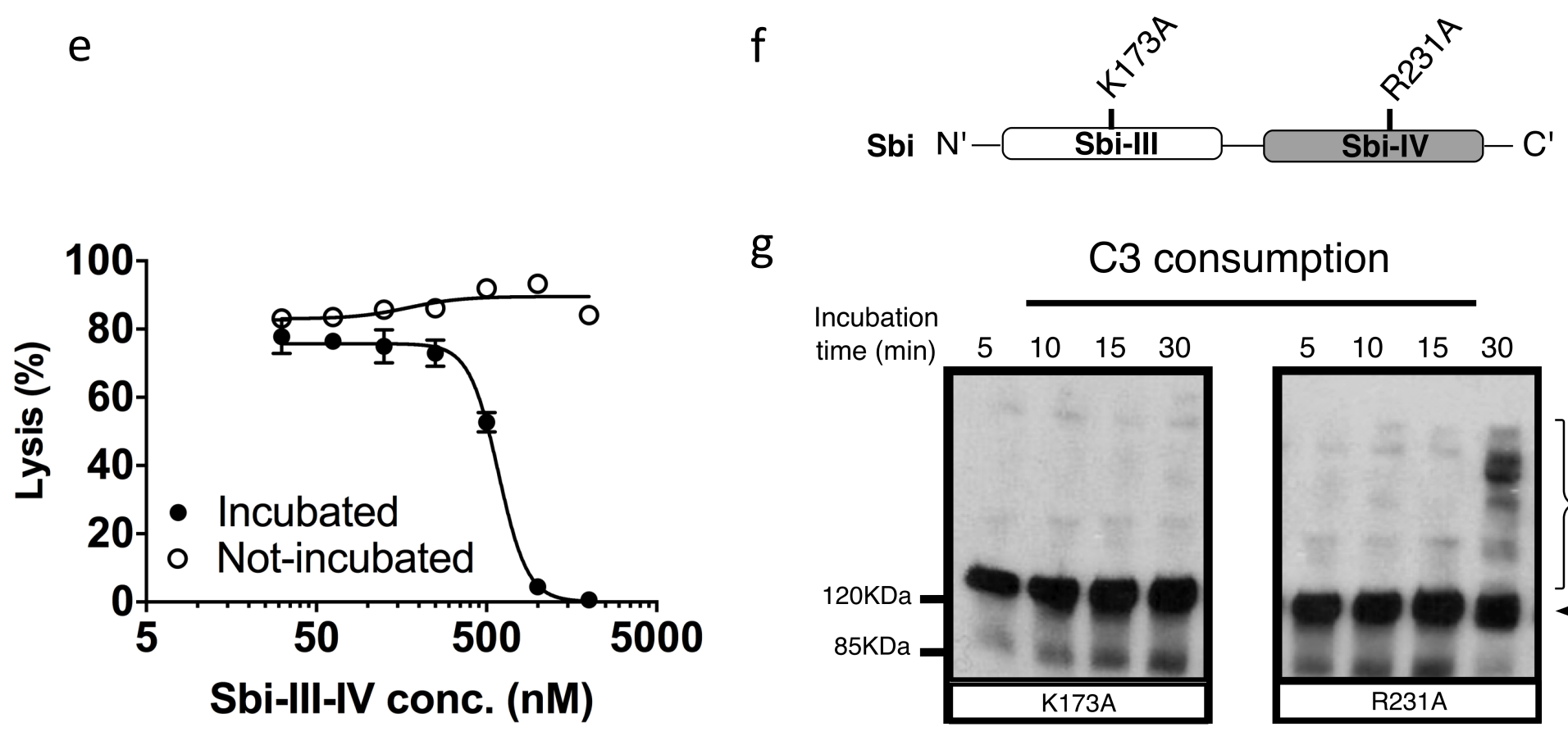

g

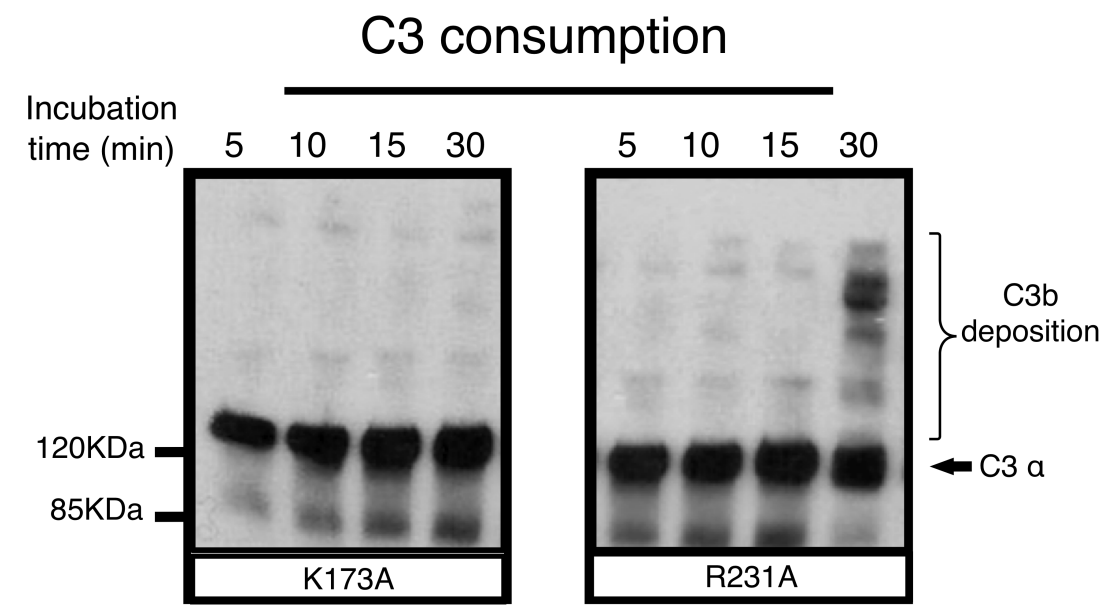


a

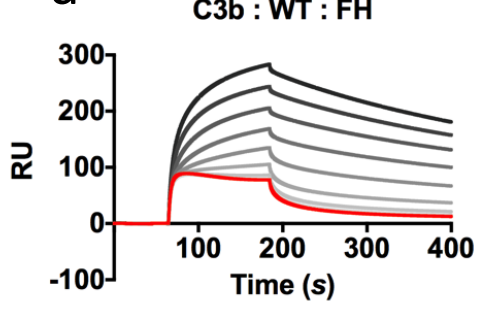

b

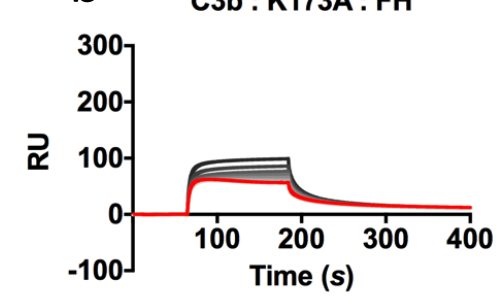

C

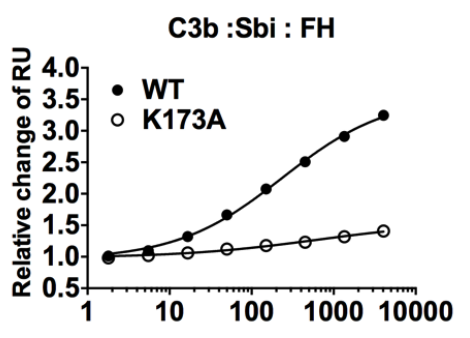

nM
C3b : WT : FHR-1

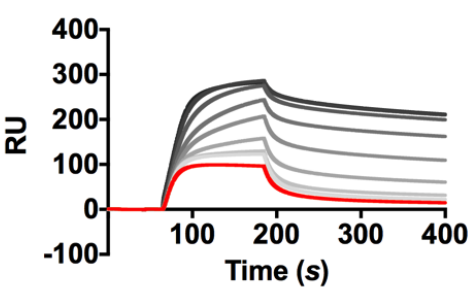

C3b : K173A : FHR-1

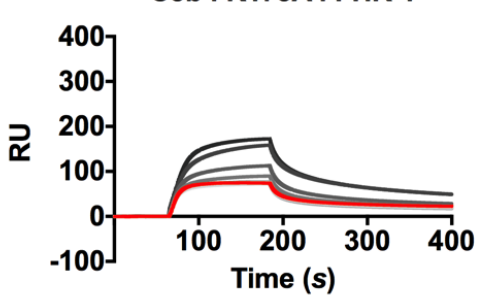

C3b :Sbi : FHR-1

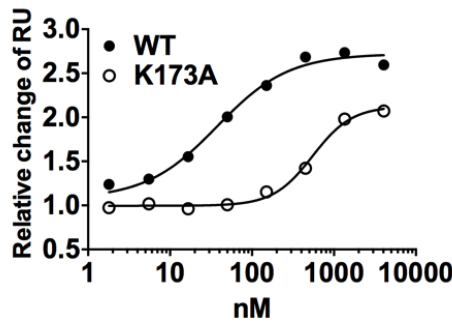

C3b : WT : FHR-2

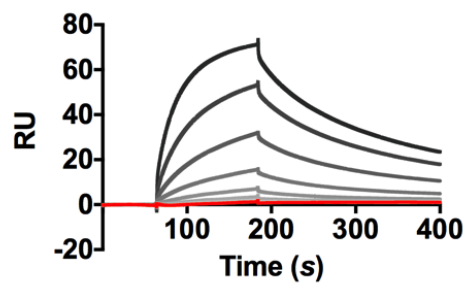

C3b : K173A : FHR-2

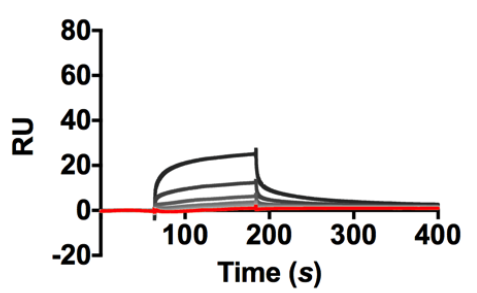

C3b : Sbi : FHR-2

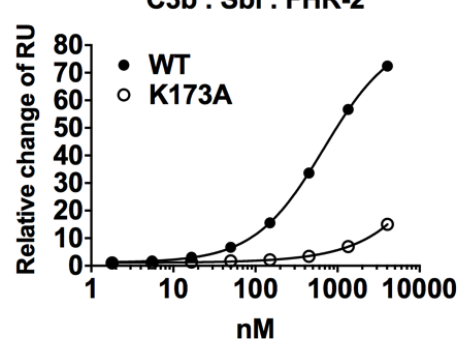

C3b : WT : FHR-5

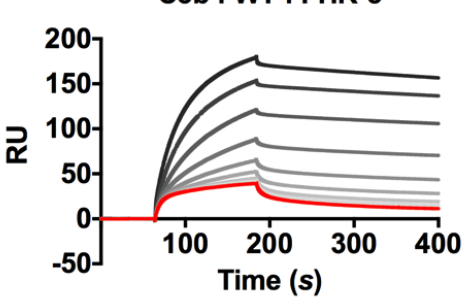

C3b : K173A : FHR-5

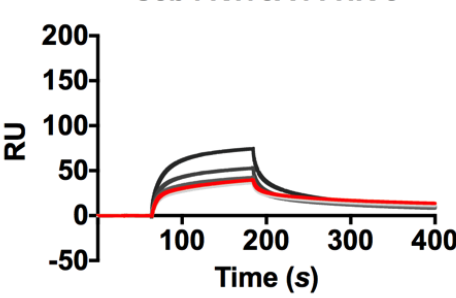

C3b : Sbi : FHR-5

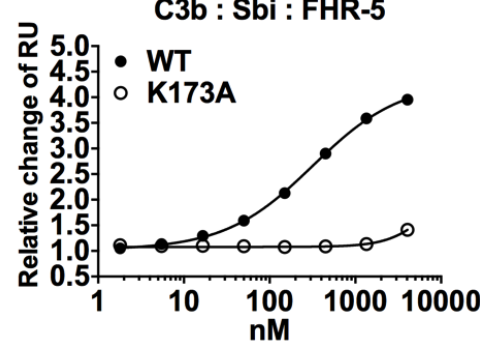

C3b : WT : $\mathrm{FH}_{19-20}$

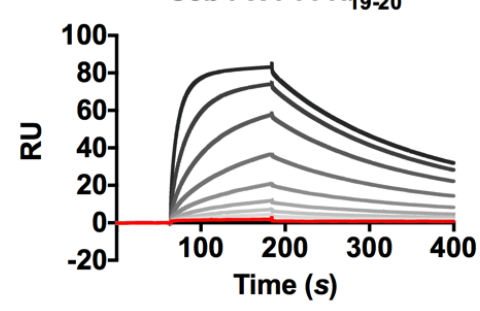

C3b : K173A : $\mathrm{FH}_{19-20}$
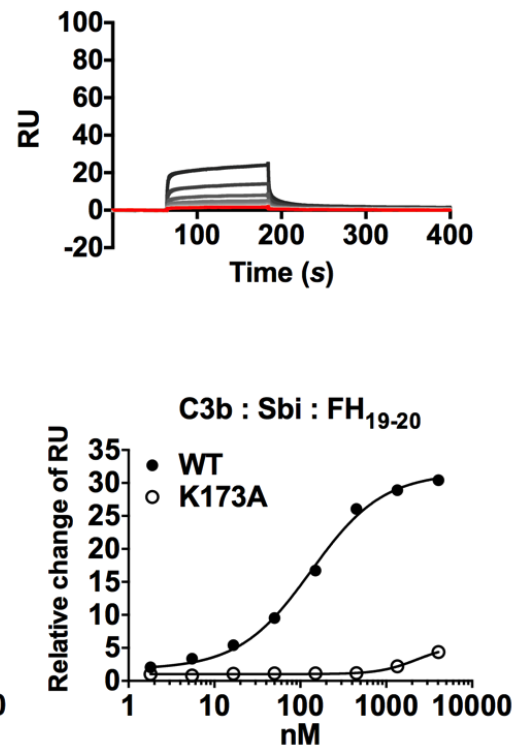
a

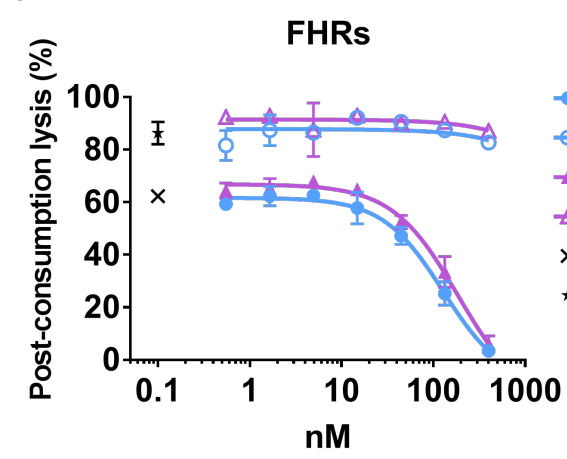

d

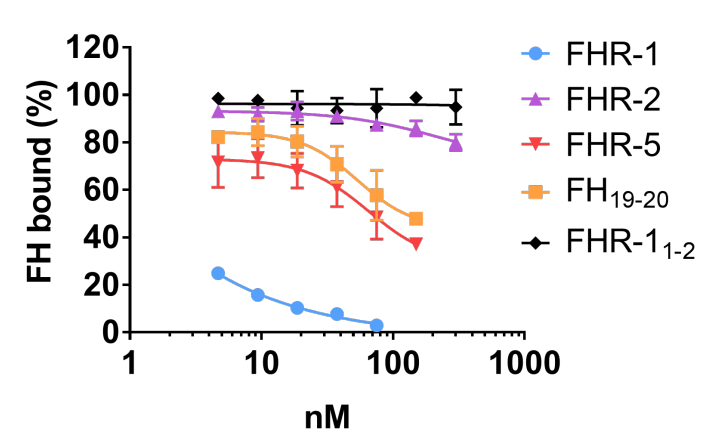

b

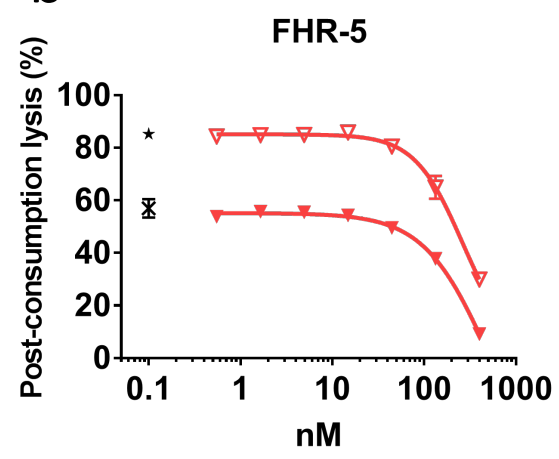

e

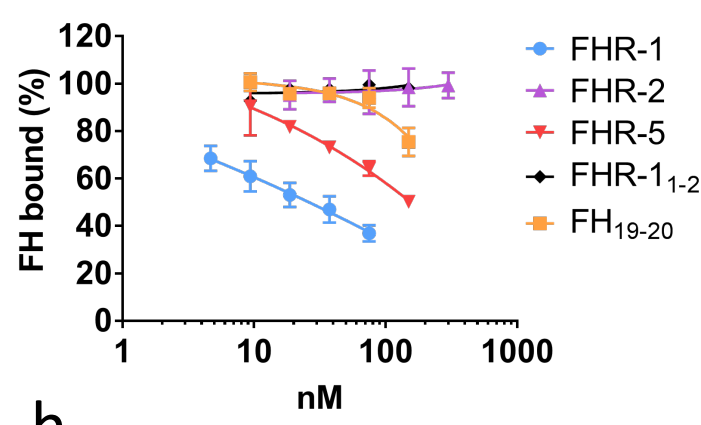

h

Sbi bound C3 convertase

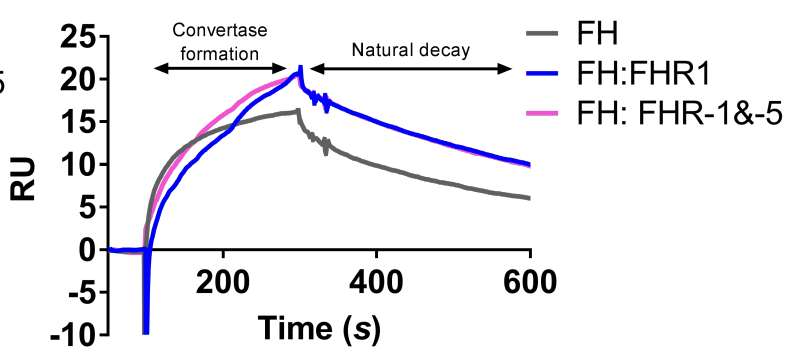

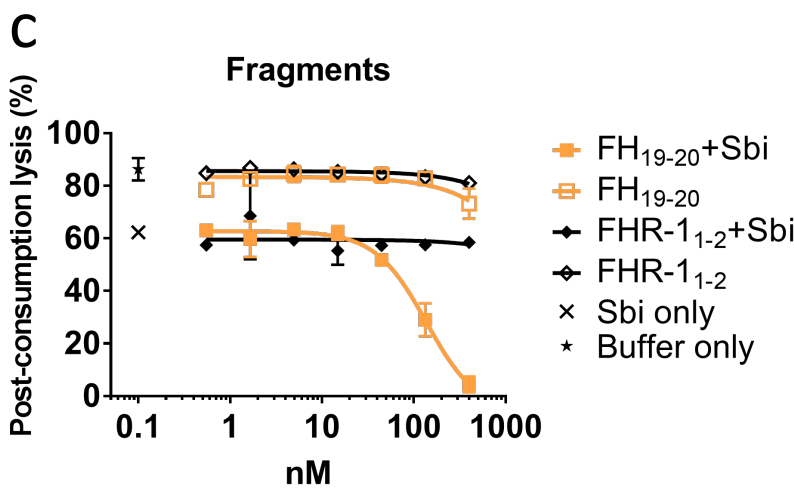

$f$

K173A
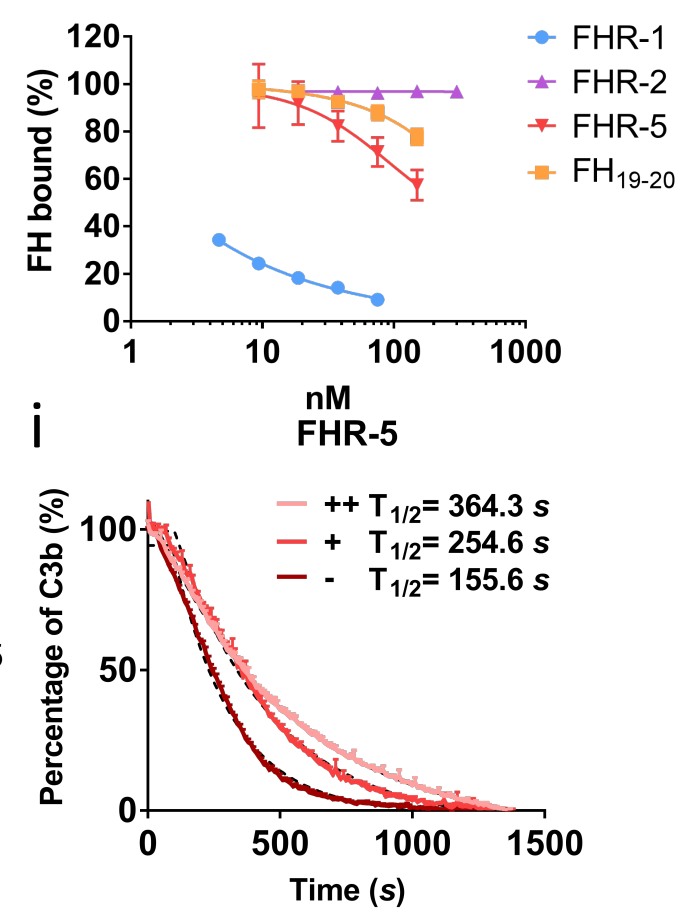

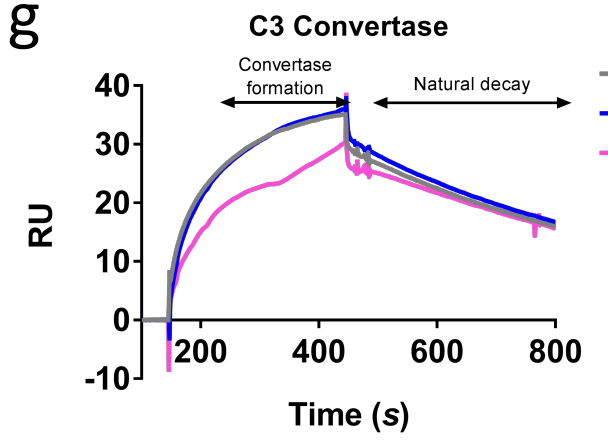

$-\mathrm{FH}$

- FH: FHR-1\&-5 


\section{$5 a$}

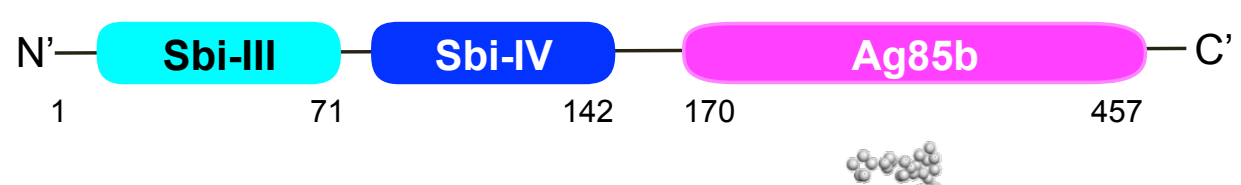

b
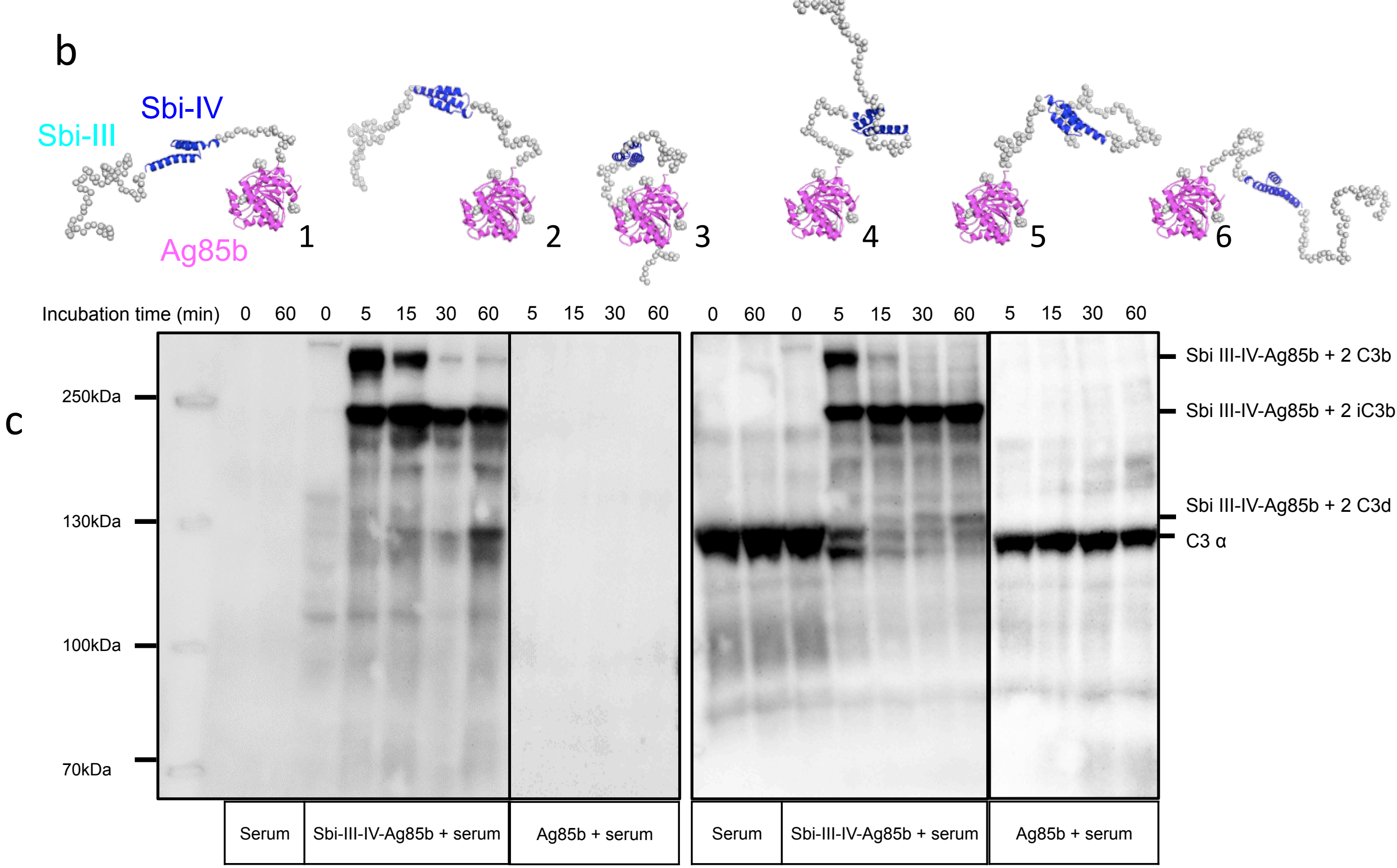

Anti-Sbi

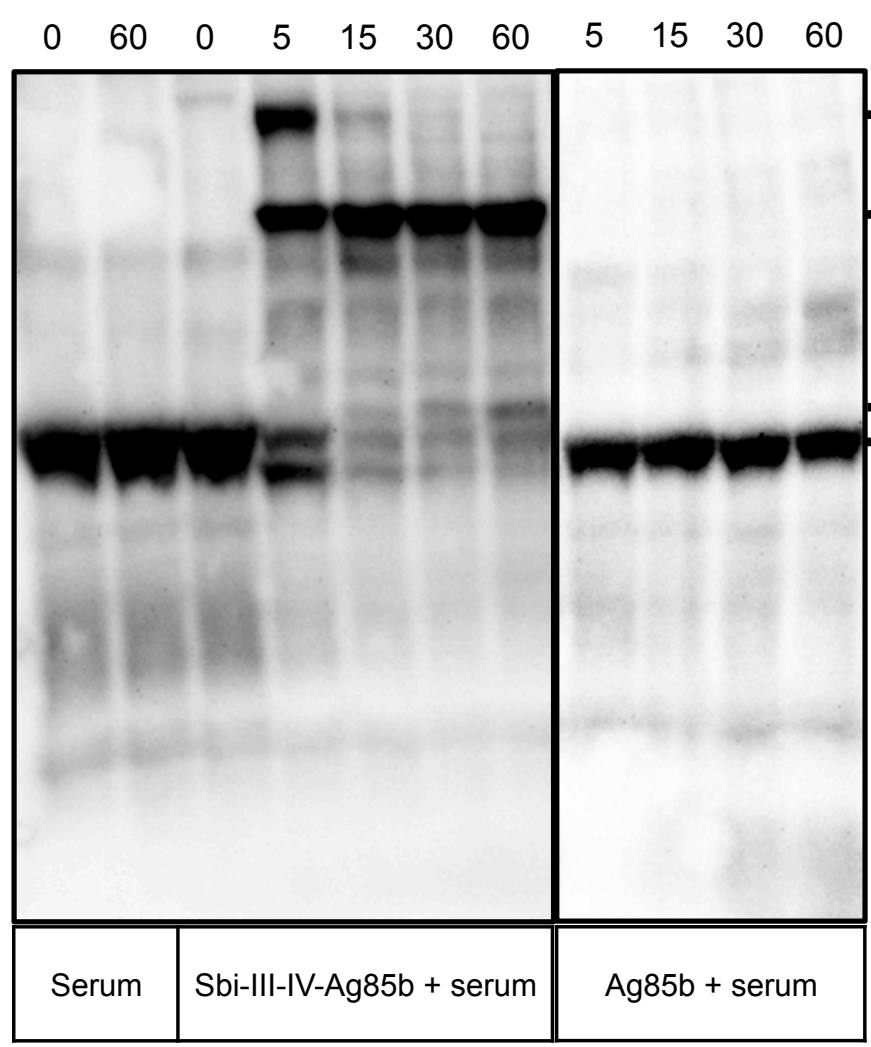

Sbi III-IV-Ag85b + 2 C3b a'

Sbi III-IV-Ag85b + 2 iC3b a'

Sbi III-IV-Ag85b + 2 C3d

C3 a 
6 a Sbi-III-IV-Ag85b | Sbi-III-IV |N | | M
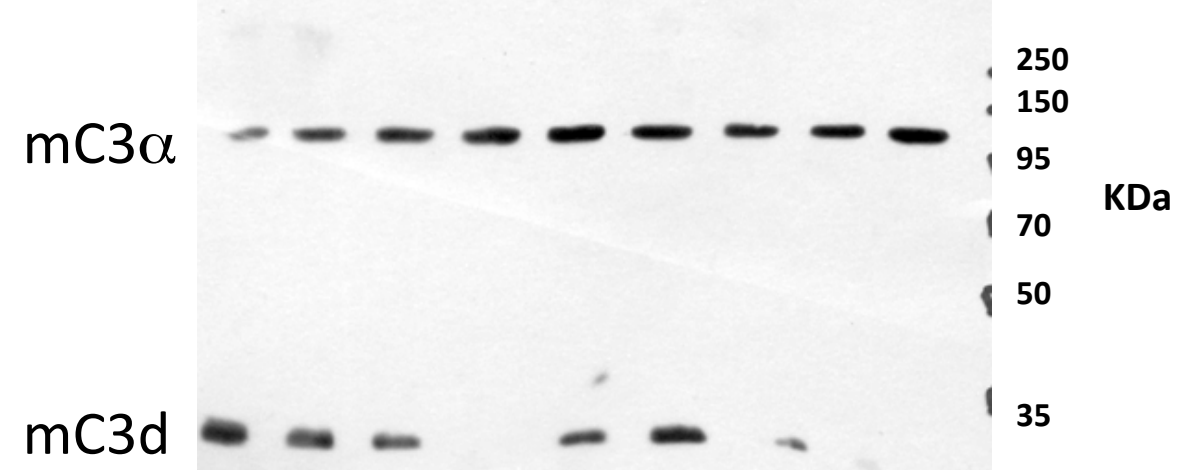

Time
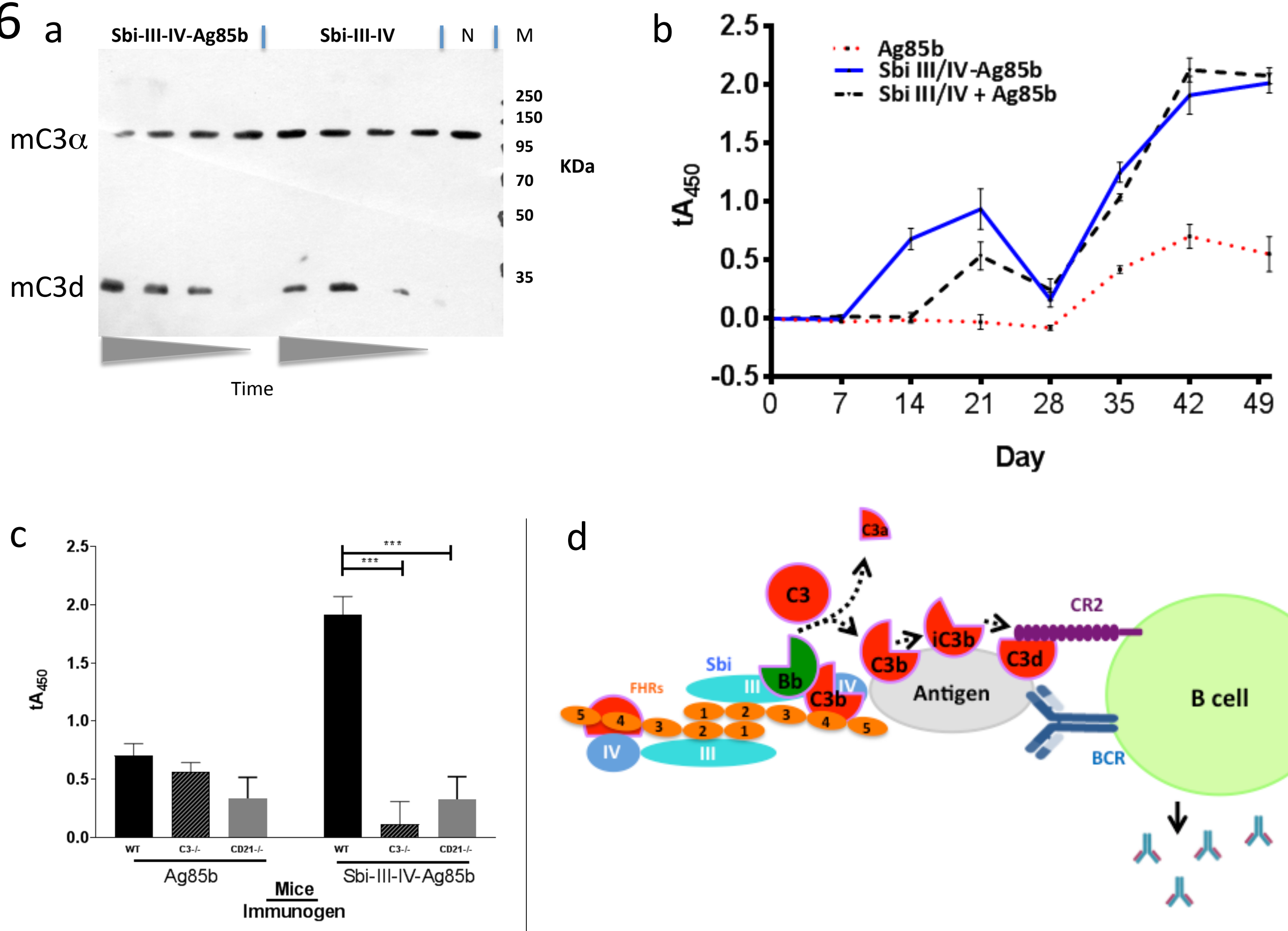
Table 1. Sbi-III-IV:C3d interaction affinity determined by SwitchSENSE

\begin{tabular}{cccc}
\hline & $\mathbf{K}_{\mathrm{d}}(\mathbf{n M})$ & $\mathbf{k}_{\mathrm{ON}}\left(\mathbf{M}^{-1} \mathbf{S}^{-1}\right)$ & $\mathbf{k}_{\text {OFF }}\left(\mathbf{S}^{-1}\right)$ \\
\hline WT:C3d & $5.0 \pm 0.8$ & $5.9 \pm 1.0 \times 10^{5}$ & $3.0 \pm 0.1 \times 10^{-3}$ \\
K173A:C3d & $5.8 \pm 1.2$ & $5.3 \pm 0.9 \times 10^{5}$ & $3.0 \pm 0.4 \times 10^{-3}$ \\
R231:C3d & & No binding \\
\hline
\end{tabular}

Table 2. Sbi-III-IV:C3d complex hydrodynamic diameter determined by SwitchSENSE

\begin{tabular}{ccc}
\hline & $\begin{array}{c}\mathbf{D}_{H}(\mathbf{n m}) \text { of } \\
\text { Sbi-III-IV }\end{array}$ & $\begin{array}{c}\mathbf{D}_{H}(\mathbf{n m}) \text { of } \\
\text { Sbi:C3d complex }\end{array}$ \\
\hline WT & $4.3 \pm 0.1$ & $6.6 \pm 0.2$ \\
K173A & $3.8 \pm 0.3$ & $5.0 \pm 0.3$ \\
R231A & $4.7 \pm 0.3$ & No binding \\
\hline
\end{tabular}

